Revue des patrimoines

\title{
Le musée de Villèle à La Réunion entre histoire et mémoire de l'esclavage. Un haut lieu de l'histoire sociale réunionnaise
}

Jean Barbier

\section{OpenEdition}

Journals

Édition électronique

URL : http://journals.openedition.org/insitu/10027

DOI : 10.4000/insitu. 10027

ISSN : 1630-7305

Éditeur

Ministère de la culture

Référence électronique

Jean Barbier, «Le musée de Villèle à La Réunion entre histoire et mémoire de l'esclavage. Un haut lieu de l'histoire sociale réunionnaise », In Situ [En ligne], 20 | 2013, mis en ligne le 11 février 2013, consulté le 30 avril 2019. URL : http://journals.openedition.org/insitu/10027 ; DOI : 10.4000/insitu.10027

Ce document a été généré automatiquement le 30 avril 2019.

\section{(c)}

In Situ Revues des patrimoines est mis à disposition selon les termes de la licence Creative Commons Attribution - Pas d'Utilisation Commerciale - Pas de Modification 4.0 International. 


\title{
Le musée de Villèle à La Réunion entre histoire et mémoire de l'esclavage. Un haut lieu de l'histoire sociale réunionnaise
}

\author{
Jean Barbier
}

1 Le musée de Villèle évoque au travers de l'histoire d'une famille de planteurs l'évolution sociale et économique d'une ancienne colonie devenue en 1946 un département français d'outre-mer.

2 Si le musée donne à voir l'histoire des maitres à partir d'un ensemble patrimonial diversifié, il restitue la mémoire de ceux qui ont travaillé sur cette propriété coloniale, d'abord les esclaves depuis le XVIII ${ }^{e}$ siècle jusqu'en 1848, puis les travailleurs engagés après l'abolition de l'esclavage jusqu'aux années 1930, et par la suite les petits colons, ouvriers agricoles liés aux propriétaires par un contrat d'affermage.

3 Aussi, au regard de l'histoire insulaire, la vocation essentielle du musée de Villèle est de donner à ses visiteurs des clés de lecture pour mieux comprendre ce qui définit la société de plantation à La Réunion et de mieux appréhender le principe fondateur sur lequel reposait à l'origine son économie, à savoir l'esclavage.

\section{Un musée insulaire : La Réunion et l'océan Indien}

4 Avant de présenter le musée historique de Villèle et les enjeux de son développement, il semble nécessaire de replacer cet équipement culturel dans son contexte insulaire, l'île de La Réunion et la zone indo-océanique, vaste espace géographique qui s'étend de l'Afrique australe jusqu'au continent australien.

5 La Réunion, anciennement appelée île Bourbon, est située au sud-est de Madagascar, au nord du Tropique du Capricorne, à environ $10000 \mathrm{~km}$ de la France métropolitaine. Elle fut 
d'abord un lieu de relâche sur la route maritime pour les navires affrétés par les Compagnies des Indes. C'est surtout aux XVIII ${ }^{\mathrm{e}}$ et $\mathrm{XIX}^{\mathrm{e}}$ siècles que s'est développée une économie de plantation. L'île devient un Département français d'outre-mer en 1946, puis une Région monodépartementale depuis les lois de décentralisation de 1982 et 1984 et enfin une des sept Régions Ultra Périphériques depuis le Traité d'Amsterdam de 1997. L'̂̂le, dont la superficie est de $2512 \mathrm{~km}^{2}$, accuse un relief très marqué avec un point culminant à $3070 \mathrm{~m}$, le Piton des Neiges (fig. $\mathbf{n}^{\circ} \mathbf{1}$ ). Elle présente des paysages très diversifiés, mais les trois quarts de sa superficie sont recouverts de zones boisées peu peuplées qui se développent à plus de $800 \mathrm{~m}$ d'altitude. Les derniers chiffres du recensement (INSEE, 2011) indiquent une population qui dépasse les 830000 habitants. Elle est jeune et offre une grande diversité culturelle. Le taux de chômage au sens du BIT (Bureau International du Travail) est très élevé et représente près de $27,2 \%$ de la population active.

Figure 1

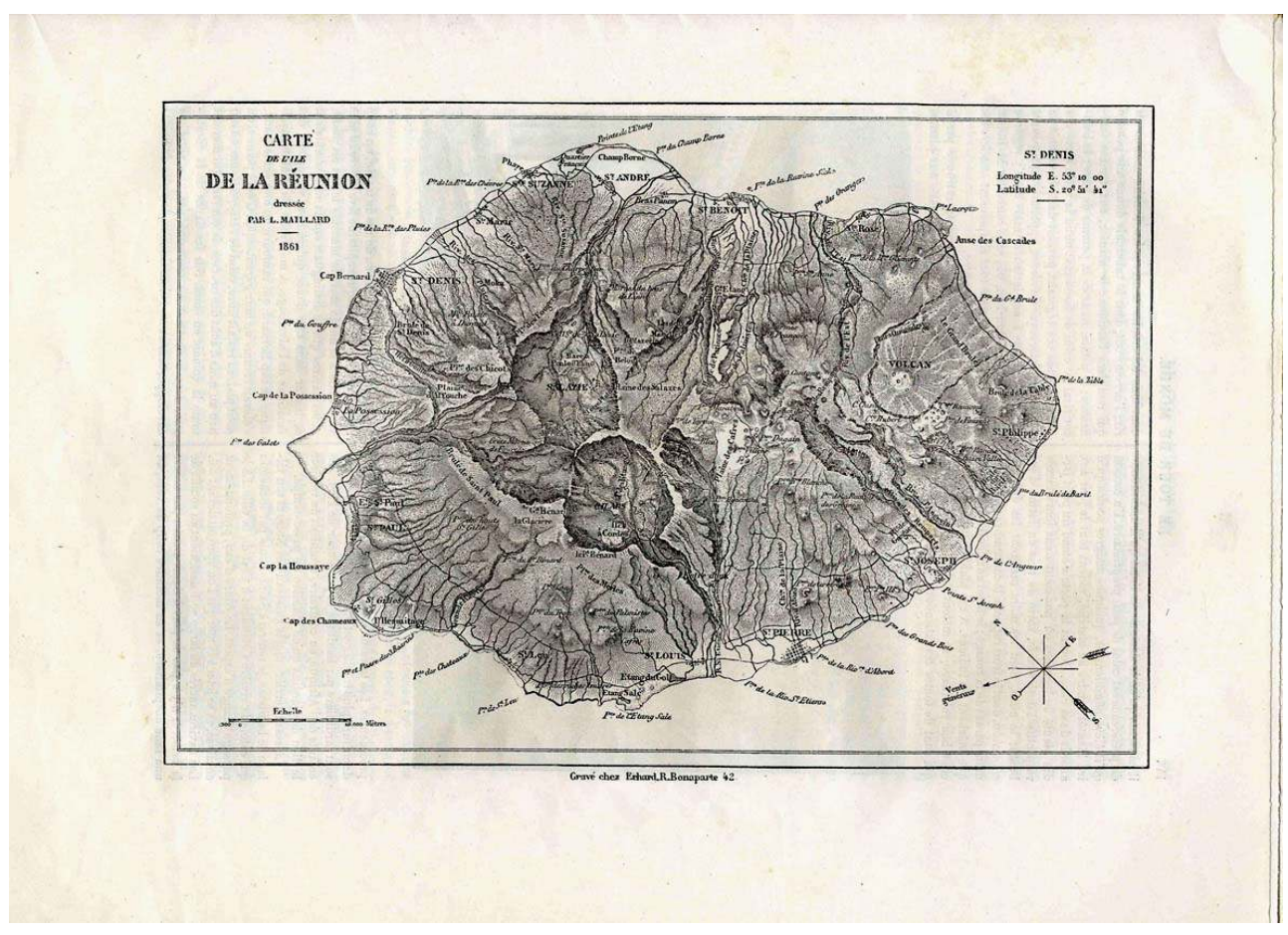

Carte de l'île de La Réunion, lithographie de 1861.

(c) Musée historique de Villèle.

6 L'île n'est peuplée d'une manière permanente qu'à partir des années 1660 et connaît plusieurs phases de peuplement. Les premiers colons français s'installent dès les années 1665 avec une main d'œuvre malgache. Avec l'ère du café et le développement d'une économie de plantation, elle connaitt une immigration depuis l'Europe, surtout des colons français, mais aussi depuis les pays de la zone indo-océanique: esclaves importés de Madagascar, d'Afrique (surtout du Mozambique) et de l'Inde. À la veille de la Révolution française, l'île compte 46000 habitants dont 35000 esclaves.

7 À partir de 1815 avec l'ère de la canne à sucre et malgré l'interdiction de la traite, des esclaves et des travailleurs engagés d'Afrique (fig. $\mathbf{n}^{\circ} 2$ ) et de l'Inde sont introduits sur 
l'île. En 1847 les esclaves représentent $56 \%$ de la population (58308 esclaves sur 103491 habitants).

\section{Figure 2}

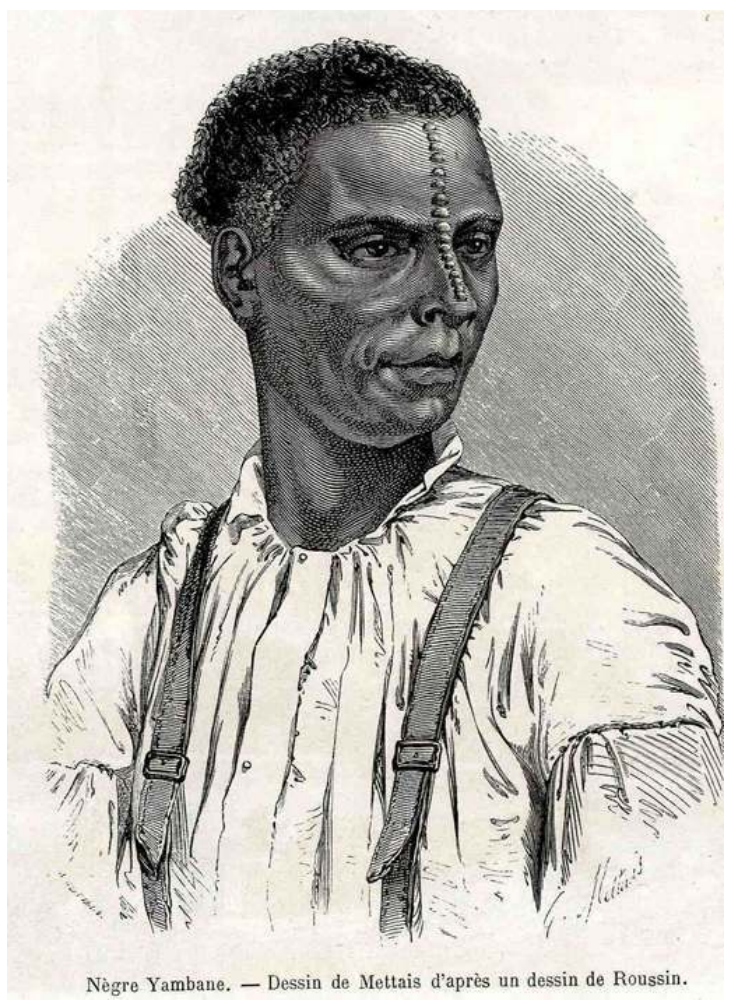

Immigrant du Mozambique, gravure de 1861.

(c) Musée historique de Villèle.

8 Après l'abolition de l'esclavage et jusque dans les années 1930, l'île fait appel à une main d'œuvre constituée par des travailleurs sous contrat ou engagés, recrutés en majorité en Inde mais aussi en Afrique, à Madagascar, en Chine, aux Comores, à Rodrigues (fig. $\mathbf{n}^{\circ} \mathbf{3}$ ). En 1881 sur les 169493 habitants de l'île, il y a entre 46000 et 62000 engagés indiens, cafres (africains), malgaches et chinois. 


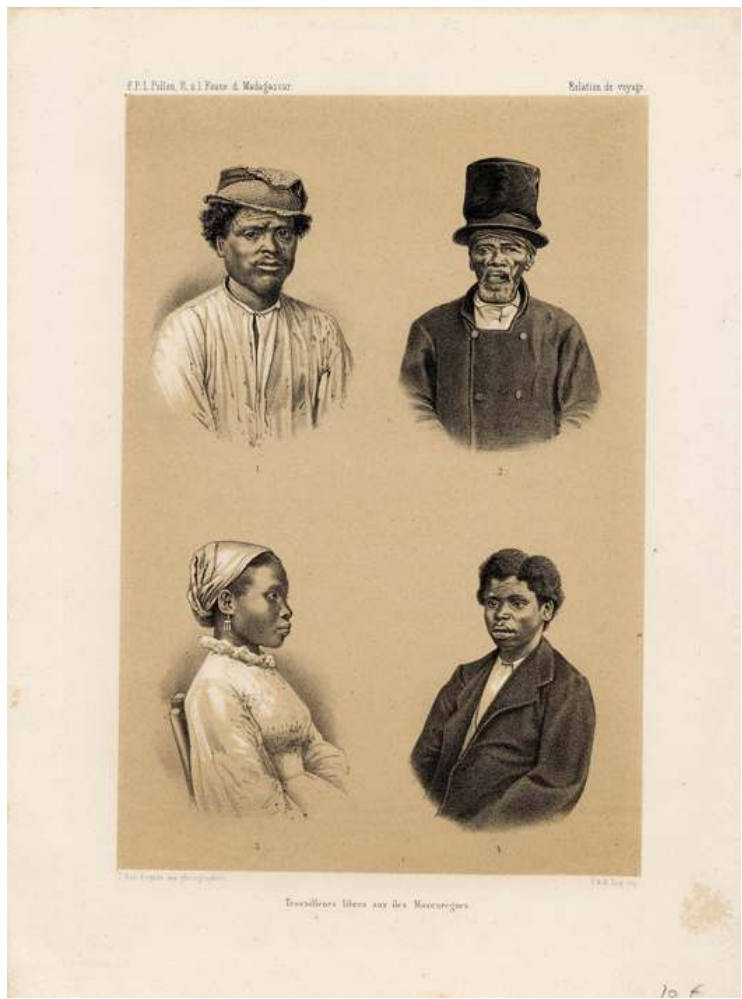

Travailleurs libres aux îles Mascareignes, lithographie du XIXe siècle.

(c) Musée historique de Villèle.

En trois siècles et demi, les différents mouvements de population vont être à l'origine de cette très grande richesse culturelle. C'est à partir de cette richesse humaine, de la coexistence de migrants d'origines diverses et de leur métissage culturel que s'est petit à petit construite l'identité de la société créole réunionnaise, identité en perpétuelle mutation.

\section{Un musée parmi d'autres : musées et lieux de mémoire à La Réunion}

Depuis les lois de décentralisation, le Département et la Région se partagent la gestion d'institutions culturelles dans le cadre de leurs compétences légales ou de compétences complémentaires, expression d'une volonté politique.

Le Département gère trois musées de France, deux d'entre eux étant situés dans le chef lieu (Saint-Denis) et un autre dans le quartier ouest de l'île ainsi qu'un lieu de mémoire implanté au nord-ouest.

Implanté à Saint-Denis au cœur du jardin de l'État, ancien jardin botanique d'acclimatation créé par la Compagnie des Indes au XVIII ${ }^{\mathrm{e}}$ siècle, le muséum d'histoire naturelle est le premier musée sur l'île. Il fut inauguré en 1855 avec pour vocation de: « donner à la jeunesse le goût de la science en général, de la zoologie et de la minéralogie en particulier ». Il était présenté à l'époque par le pouvoir colonial comme : « la sentinelle de la pensée française dans la mer des Indes ». À l'origine musée d'État, il passe sous la 
tutelle administrative du Conseil général en 1992. Depuis 2007, le muséum assure la gestion scientifique d'une annexe muséale ouverte dans les anciennes salines situées sur la commune de Saint-Leu.

Le musée Léon Dierx est créé également à Saint-Denis par la Colonie en 1911, à l'initiative de deux écrivains insulaires Marius et Ary Leblond (pseudonymes de Georges Athénas et Aimé Merlo) afin d'offrir un «lieu d'éducation et de mémoire" à La Réunion, île considérée à l'époque par le pouvoir colonial comme "la petite France de l'océan Indien ». Des collections d'art moderne sont constituées sous l'égide d'un comité parisien composé d'artistes, de marchands d'art et d'intellectuels et, à La Réunion, des dons de collections d'art local et d'histoire sont effectués. Le musée Léon Dierx devient essentiellement un musée d'art à partir de 1947 avec l'arrivée du don de Lucien Vollard (frère du célèbre marchand Ambroise) comprenant 157 œuvres d'art représentatives des différents mouvements de l'art moderne en France.

Le musée historique de Villèle est créé en 1974 près de trente ans après la départementalisation afin de sauvegarder les témoignages architecturaux d'une propriété coloniale située sur la commune de Saint-Paul (fig. $\left.\mathbf{n}^{\circ} \mathbf{4}\right)$ et de doter l'île de La Réunion d'un musée historique. Depuis 2007 un lieu de mémoire situé au lieu-dit La Grande Chaloupe a été rattaché à ce musée. Il est constitué d'anciens lazarets, lieux de quarantaine bâtis à partir des années 1860 pour protéger l'île des épidémies et accueillir les nombreux engagés introduits à La Réunion. Ce site patrimonial fait depuis plusieurs années l'objet d'un travail de restauration et se présente comme le lieu emblématique de l'histoire du peuplement à La Réunion.

Figure 4

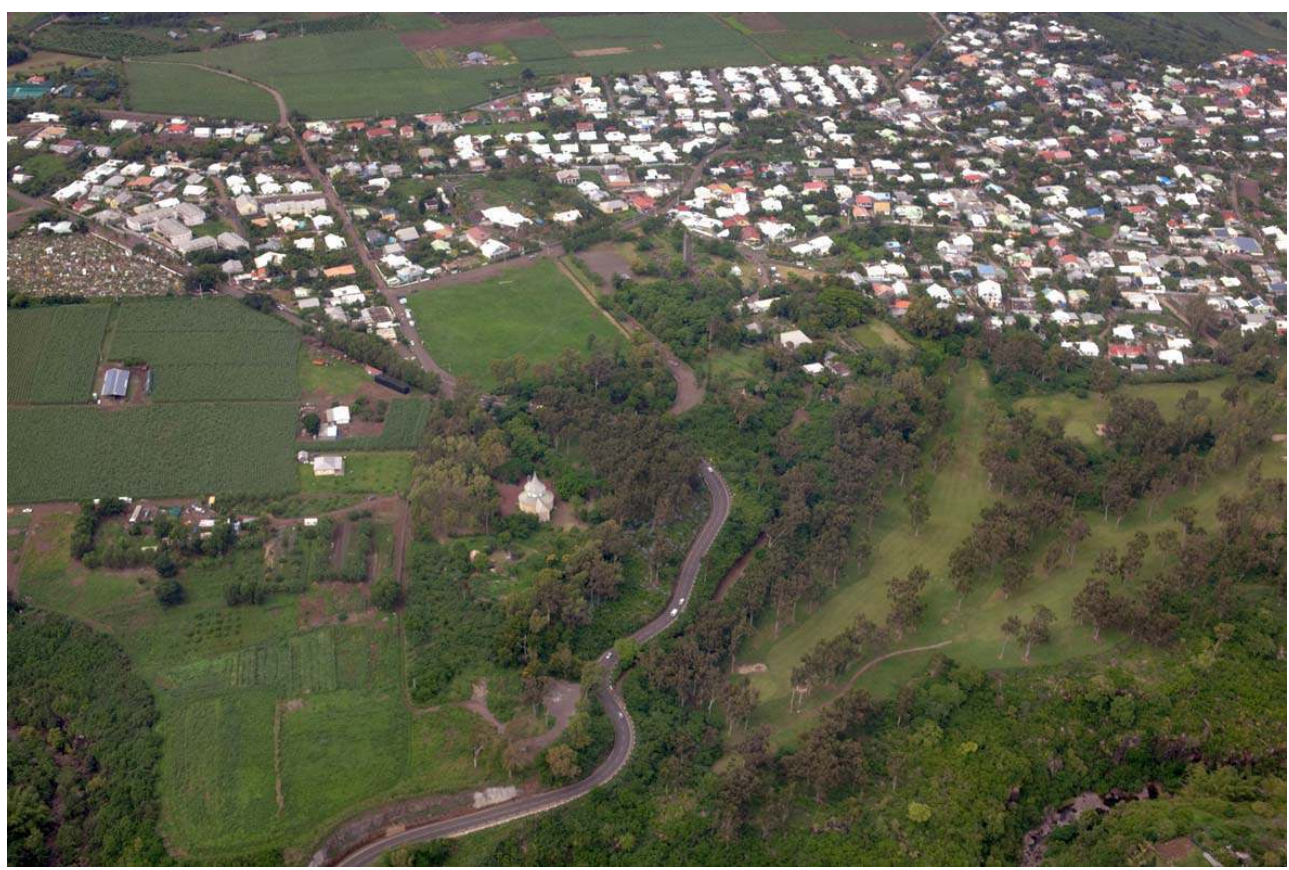

Vue aérienne du musée historique et du lieu-dit « Villèle », emplacement de l'ancien camp d'esclaves, photographie en 2011

(c) Musée historique de Villèle / Raymond Barthes.

15 La Région gère quant à elle deux musées de France ainsi que deux centres de recherche scientifique. 
Créé au début des années 1990 dans une ancienne usine à sucre sur la commune de SaintLeu (ouest de l'île), le musée Stella Matutina a pour vocation l'histoire du développement industriel de l'île et notamment l'histoire du sucre. Ce musée s'est toutefois orienté depuis ces dernières années vers un nouveau concept, en recentrant sa vocation sur la mémoire des hommes et du sucre.

17 En gestation depuis de nombreuses années, un musée des arts décoratifs de l'océan Indien - le MADOI - a été inauguré en 2007 sur la commune de Saint-Louis (sud de l'île).

La Maison du Volcan créée en 1992 sur la commune du Tampon à proximité du Piton de la Fournaise - volcan toujours en activité - et le centre Kélonia situé à Saint-Leu, inauguré en 2006 et consacré à l'étude des tortues marines, représentent deux centres scientifiques très attractifs pour le tourisme.

19 Un projet muséal a été abandonné. En effet, les élections régionales de mai 2010 ont sonné le glas de la Maison des Civilisations et de l'Unité Réunionnaise - MCUR -, projet culturel ambitieux porté par l'ancienne mandature de la Région et qui était le faire-valoir de sa politique culturelle. À l'image des grands projets muséographiques internationaux initiés ces dix dernières années, le projet réunionnais était très novateur dans sa conception et dans sa traduction architecturale. Il se présentait à la fois comme un musée de société et un musée d'histoire tout en étant un espace scientifique et culturel.

\section{La vocation historique du musée de Villèle}

20 (fig. $\left.\mathbf{n}^{\circ} 5\right)$

21 Le musée de Villèle est avant tout un lieu d'histoire évoquant, à partir des traces matérielles encore existantes, l'évolution et les transformations d'une propriété coloniale sur une période qui couvre presque deux siècles. Il met en scène l'histoire de protagonistes que la logique du système colonial oppose : d'un côté les maîtres, colons immigrants ou descendants de colons originaires de l'Europe et plus précisément de la France, de l'autre la population servile, quantitativement plus nombreuse et constituée d'esclaves ou d'engagés venus de Madagascar, d'Afrique orientale, ou d'Asie. 
Figure 5

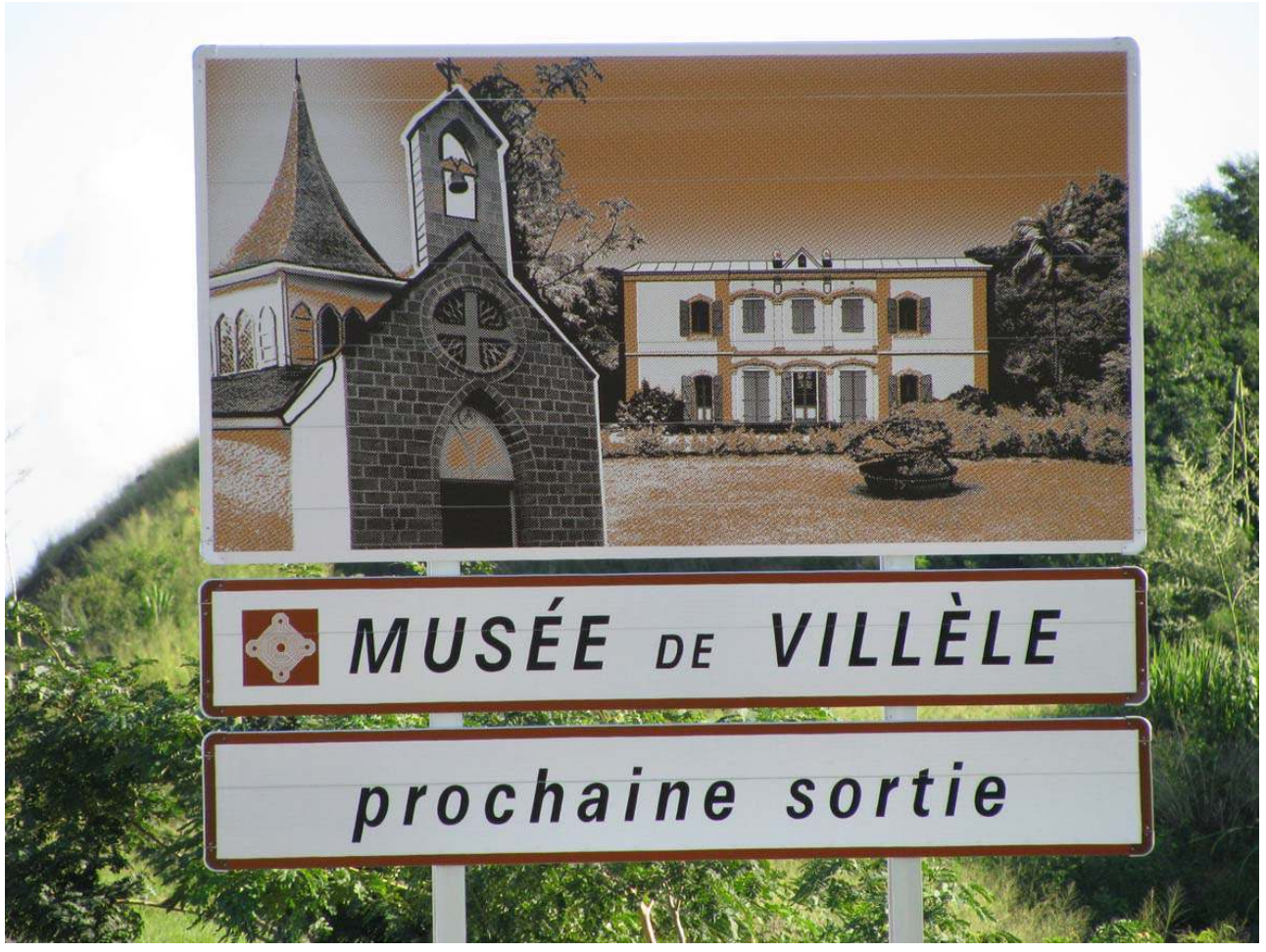

Panneau directionnel sur la route des Tamarins, photographie en 2011.

(c) Musée historique de Villèle / Jean Barbier.

22 L'ancienne habitation Desbassayns (fig. $\mathbf{n}^{\circ} \mathbf{6}$ ) - terme en usage aux XVIII ${ }^{e}$ et XIX ${ }^{e}$ siècles pour désigner la propriété du planteur -, offre toutes les caractéristiques pour définir et comprendre le système de l'économie de plantation à La Réunion, que ce soit en termes de mode de production et d'exploitation d'une unité foncière ou d'instrumentalisation d'une population servile. 
Figure 6

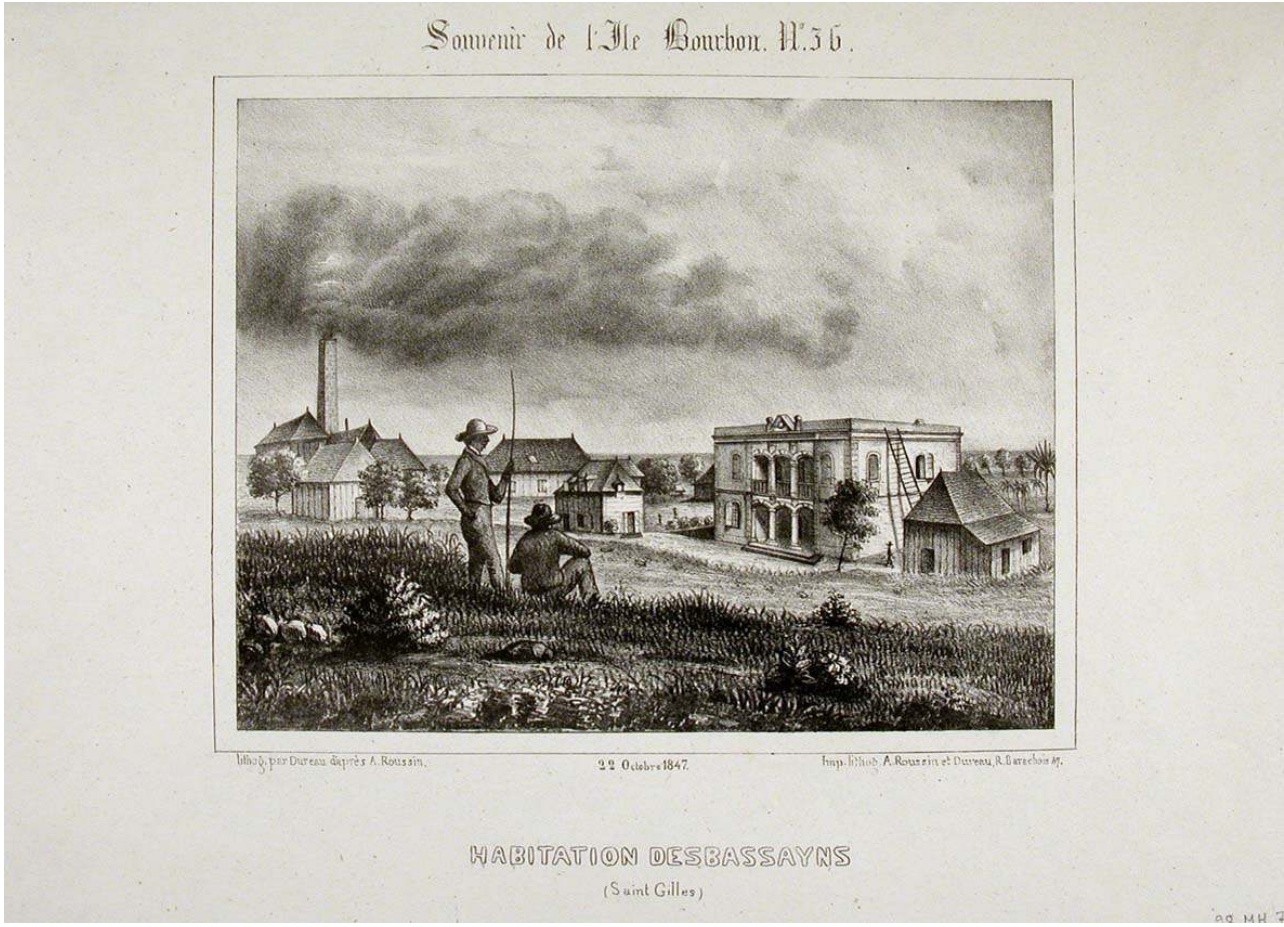

Habitation Desbassayns, lithographie de 1847.

(c) Musée historique de Villèle.

\section{Un lieu d'histoire}

23 Situé à Saint-Gilles-les-Hauts, ce domaine est établi durant la seconde moitié du XVIII siècle à partir de plusieurs concessions délivrées au XVII ${ }^{\mathrm{e}}$ siècle et réunies par la volonté d'une riche famille de colons créoles, les Panon-Desbassayns. Henri Paulin (fig. $\mathbf{n}^{\circ}$ ) ) et Ombline Panon-Desbassayns, héritiers l'un et l'autre d'un important patrimoine foncier, n'ont de cesse d'accroître leur patrimoine commun et d'étendre les limites de leur propriété qui se développe en lanière depuis la côte au-delà des pas géométriques ${ }^{1}$ et des communes jusque dans les Hauts du quartier de Saint-Paul, au niveau de la ligne domaniale située à environ 1400 m d'altitude. 
Figure 7

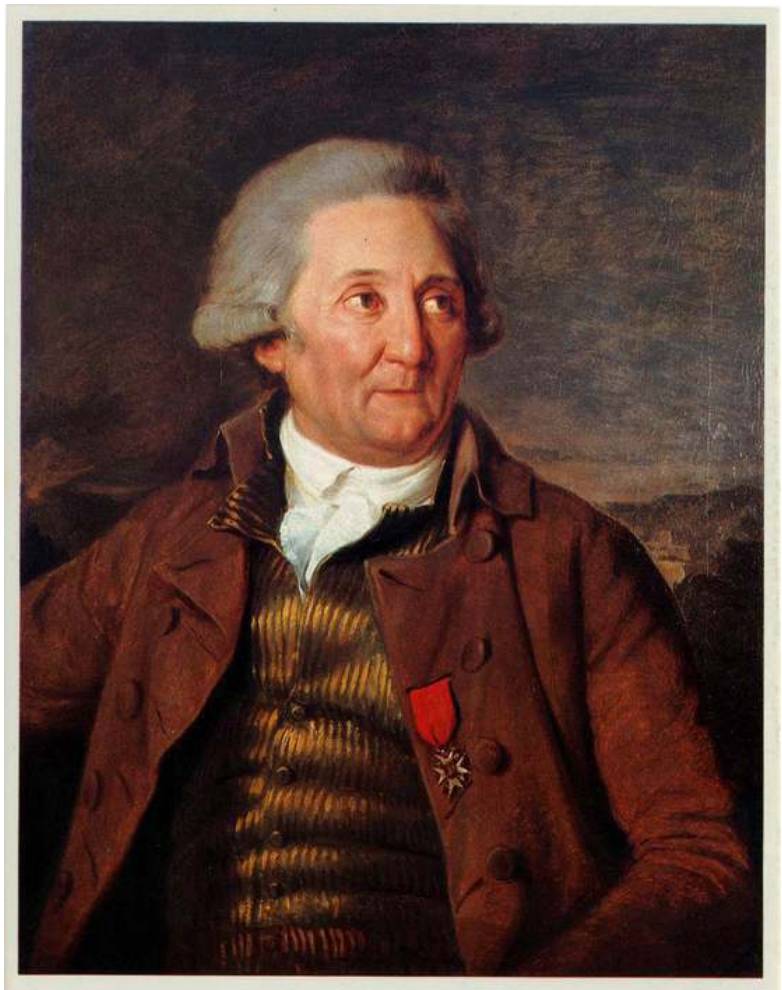

Henri Paulin Panon Desbassayns (1732-1800), huile sur toile, 1792.

(c) Musée historique de Villèle / Daniel Auguste.

La prospérité de l'habitation repose sur l'exploitation des terres en faire-valoir direct, production de coton et surtout de café au XVIII ${ }^{\mathrm{e}}$ siècle supplantée par la culture de la canne à sucre à partir du premier tiers du XIX siècle.

La mise en valeur des terres nécessite le recours à une main d'œuvre servile, constituée essentiellement d'esclaves jusqu'en 1848, Africains (ou Cafres), Malgaches, Indiens (sousreprésentés) mais aussi des créoles (nés sur l'île) puis, après l'abolition, de travailleurs sous contrat appelés engagés, originaires également de l'Afrique, de Madagascar mais surtout de l'Inde.

En 1845 la famille Desbassayns possédait deux habitations à Saint-Paul représentant 492 hectares de surfaces cultivées et une force de travail de 401 esclaves.

Après la disparition de la veuve Desbassayns le 4 février 1846, le domaine passa entre les mains de plusieurs de ses enfants pour revenir à Céline, l'une de ses petites filles mariée à son cousin Frédéric de Villèle, neveu du ministre des finances sous la Restauration, Joseph de Villèle, qui lui-même avait épousé à Bourbon en 1799 Mélanie Panon Desbassayns.

Avec la crise qui affecte l'économie sucrière dès la fin du XIX siècle et qui s'intensifie durant la première moitié du siècle suivant, afin de conserver l'unité foncière du domaine, les héritiers de la famille Villèle se regroupent en 1927 (fig. $\mathbf{n}^{\circ} \mathbf{8}$ ) au sein d'une société anonyme. Ils ont recours à l'emprunt pour investir en matériel et améliorer les capacités de production de sucre de canne et passer à un autre mode de gestion, le colonat partiaire fondé sur le principe d'une exploitation indirecte des terres : affermage instituant $1 / 3$ des revenus aux propriétaires contre $2 / 3$ aux colons. 
Figure 8

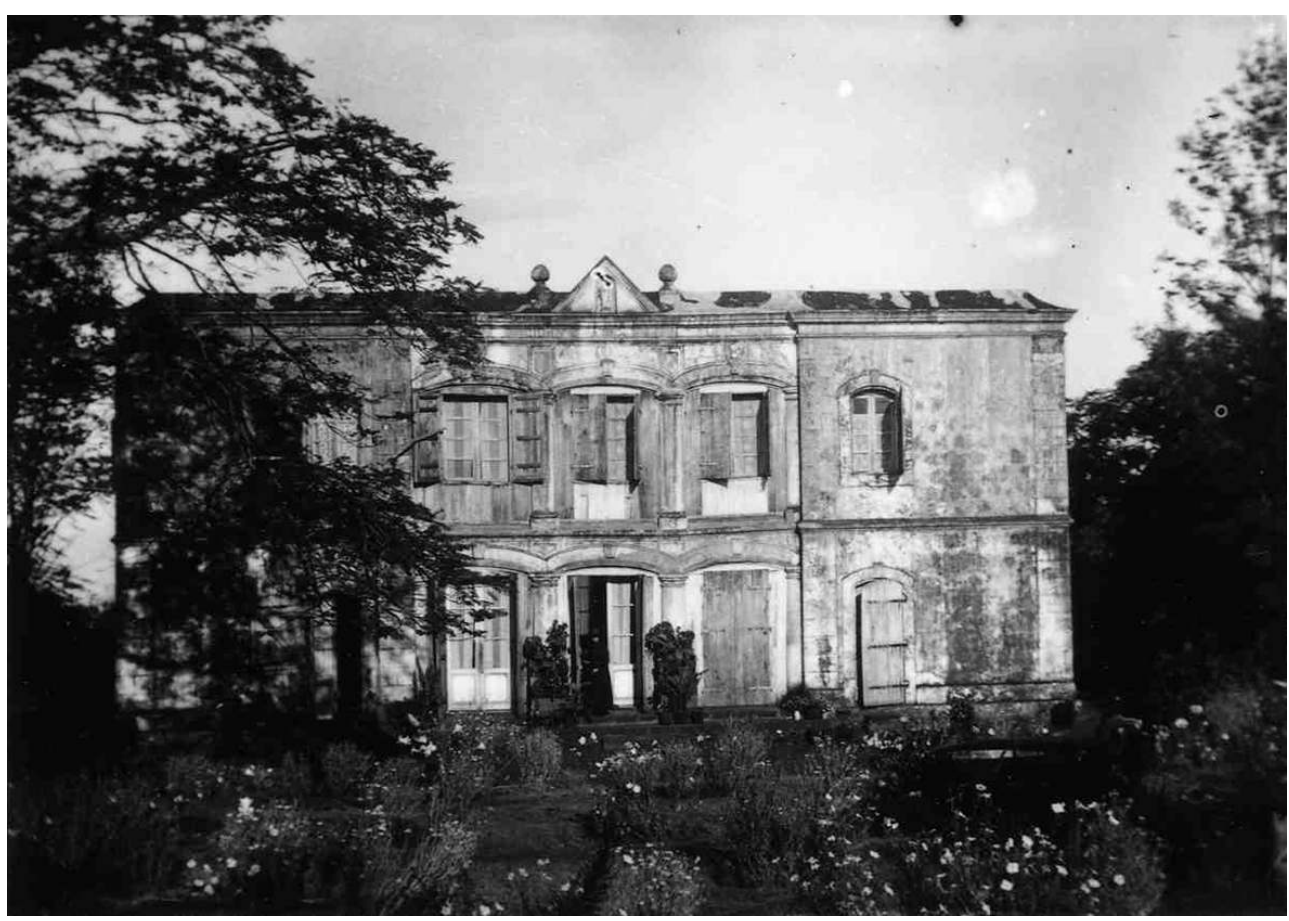

Maison Desbassayns, aujourd'hui musée historique de Villèle, photographie vers 1920

(c) Musée historique de Villèle.

29 Finalement, la propriété est cédée en 1960 à une société de crédit, le Crédit Foncier Colonial et les derniers descendants gardent la jouissance de la maison familiale au terme d'un bail à loyer pour 30 ans signé en 1971. Deux années après leur départ définitif en France hexagonale, une page de l'histoire des grands domaines sucriers se tourne et marque la fin de la dynastie Desbassayns-Villèle.

30 Afin de conserver ce remarquable ensemble patrimonial et d'y ouvrir un musée historique, une partie de la propriété - dont la maison de maître - est cédée au Conseil général de La Réunion en 1973 par le conseil d'administration de la société propriétaire. Le musée historique de Saint-Gilles-les-Hauts est créé en 1974 et inauguré en 1976.

\section{Un lieu de mémoire}

Le musée se présente aussi comme un lieu d'interprétation de l'esclavage au travers de la figure emblématique d'un personnage tout à fait exceptionnel qui a marqué l'inconscient collectif de la société réunionnaise, Madame Desbassayns (fig. $\left.\mathbf{n}^{\circ} \mathbf{9}\right)$. Cette femme créole a dirigé seule et de main de fer pendant plus de quarante-six ans, de 1800 (date de disparition de son mari) jusqu'à 1846, ses deux plantations situées dans le quartier de Saint-Paul où vivaient et travaillaient plus de quatre cents esclaves. 
Figure 9

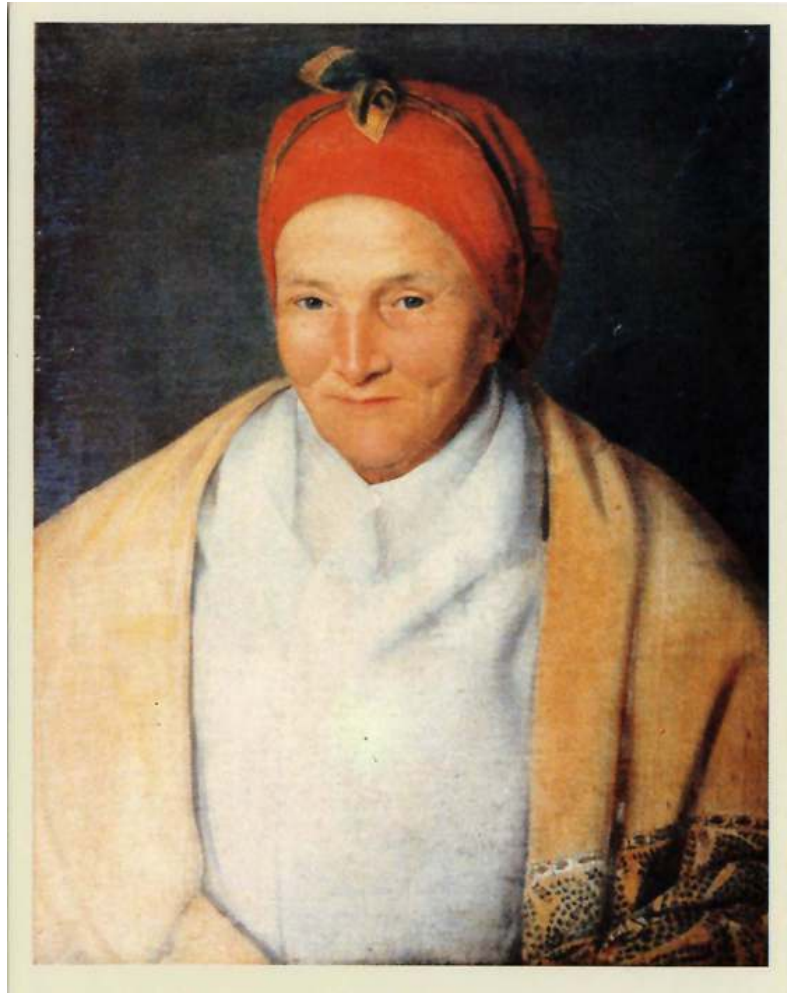

Ombline Panon-Desbassayns (1755-1846), huile sur toile, XIXe siècle.

Collection particulière. ( Musée historique de Villèle / Daniel Auguste.

Le personnage de Madame Desbassayns, figure historique peu ordinaire et très controversée, présente un double aspect où s'opposent les antagonismes de la société réunionnaise. Elle apparaît en effet sous deux représentations antithétiques, mi-ange, midémon, à la fois la Seconde Providence qui administra son domaine avec fermeté selon un modèle paternaliste et sous les traits de granmèr Kal (figure populaire des contes créoles derrière laquelle se cache une sorcière) à qui l'on prête les crimes les plus abominables. Au fil du temps, les représentations symboliques de Madame Desbassayns ont évolué au gré des transformations sociales et politiques de La Réunion, cristallisant le malaise et les peurs de la société post- coloniale.

Le musée de Villèle concilie une approche historique de ce personnage inscrit dans la réalité sociale, politique et religieuse de son époque et prend en compte également ce qu'est devenue Madame Desbassayns dans l'imaginaire réunionnais, une sorte de monstre chimérique parangon du système esclavagiste.

Dans la petite chapelle domestique (fig. $\mathbf{n}^{\circ} \mathbf{1 0}$ ) située à proximité de la maison principale se trouve la plaque tombale de Madame Desbassayns mise en place le 4 février 1866 lors du transfert de ses restes depuis le lieu d'inhumation initial dans le cimetière de la ville de Saint-Paul. Si l'épitaphe gravée sur le marbre de la dalle évoque «la Seconde Providence ", dans l'imaginaire populaire, la pierre brisée par le cyclone du 4 février 1932 - date anniversaire de la mort de la défunte - révèle les preuves matérielles, véritables stigmates de son infamie. 


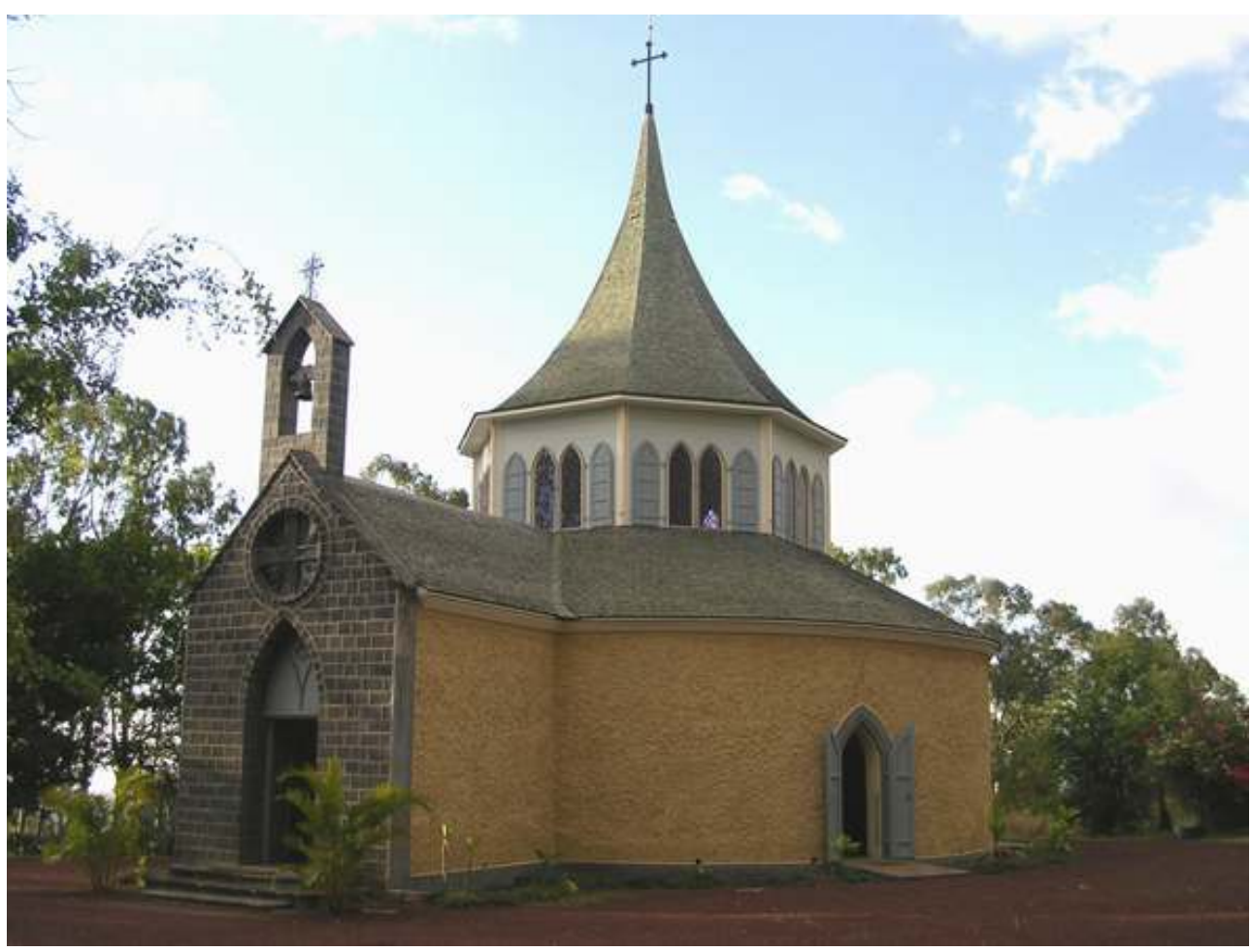

Chapelle Pointue, photographie en 2010.

(c) Musée historique de Villèle / Raymond Barthes.

À l'évidence, au cours du temps, la réputation de la Seconde Providence a « pris du plomb dans l'aile» et aujourd'hui, pour de nombreux Réunionnais, prononcer le nom Desbassayns revient à dénoncer la dureté du système colonial et le goût amer d'une époque révolue mais à tout jamais marquée par le drame de l'esclavage.

\section{Un ensemble patrimonial diversifié}

\section{Les bâtiments}

Cet ensemble affirme sa vocation patrimoniale de différentes manières.

En premier lieu, au travers de l'histoire de ses murs, à partir des traces bien visibles, en d'autres termes ce qu'il donne à voir avec les bâtiments anciens conservés in situ et qui témoignent de l'organisation sociale, économique et religieuse d'une propriété coloniale.

La maison de maître achevée en 1788 reprend un modèle d'architecture classique importée de Pondichéry (fig. $\mathbf{n}^{\circ} \mathbf{1 1}$ ). Elle est décrite au XIX ${ }^{\mathrm{e}}$ siècle comme : " un château d'architecture malabare ». C'est cette demeure qui est l'espace muséal principal. Les salles se développent sur deux niveaux. Les sept pièces du rez-de-chaussée présentent les collections permanentes et les quatre salles situées à l'étage sont dévolues à la présentation d'expositions temporaires. 
Figure 11

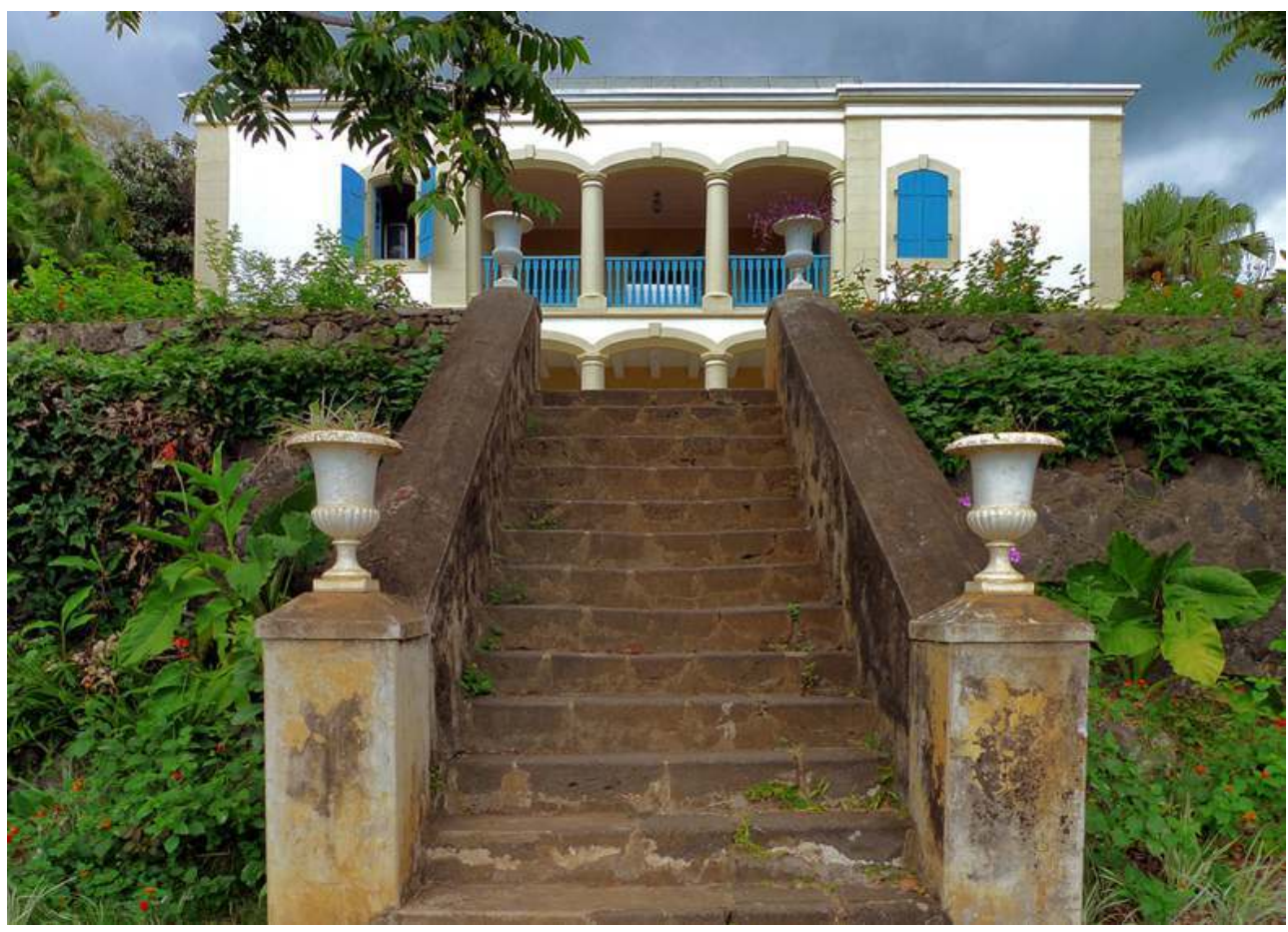

Musée historique, façade occidentale, photographie en 2010.

c C Musée historique de Villèle / Raymond Barthes.

39 La cuisine des maîtres (fig. $\mathbf{n}^{\circ} \mathbf{1 2}$ ) occupe une partie d'un bâtiment annexe situé à proximité de la maison principale, respectant en cela la tradition créole. Dans le testament olographe de Madame Desbassayns rédigé en 1845, est mentionnée également une cuisine pour les Noirs, bâtiment non conservé et non localisé à ce jour ! 
Figure 12

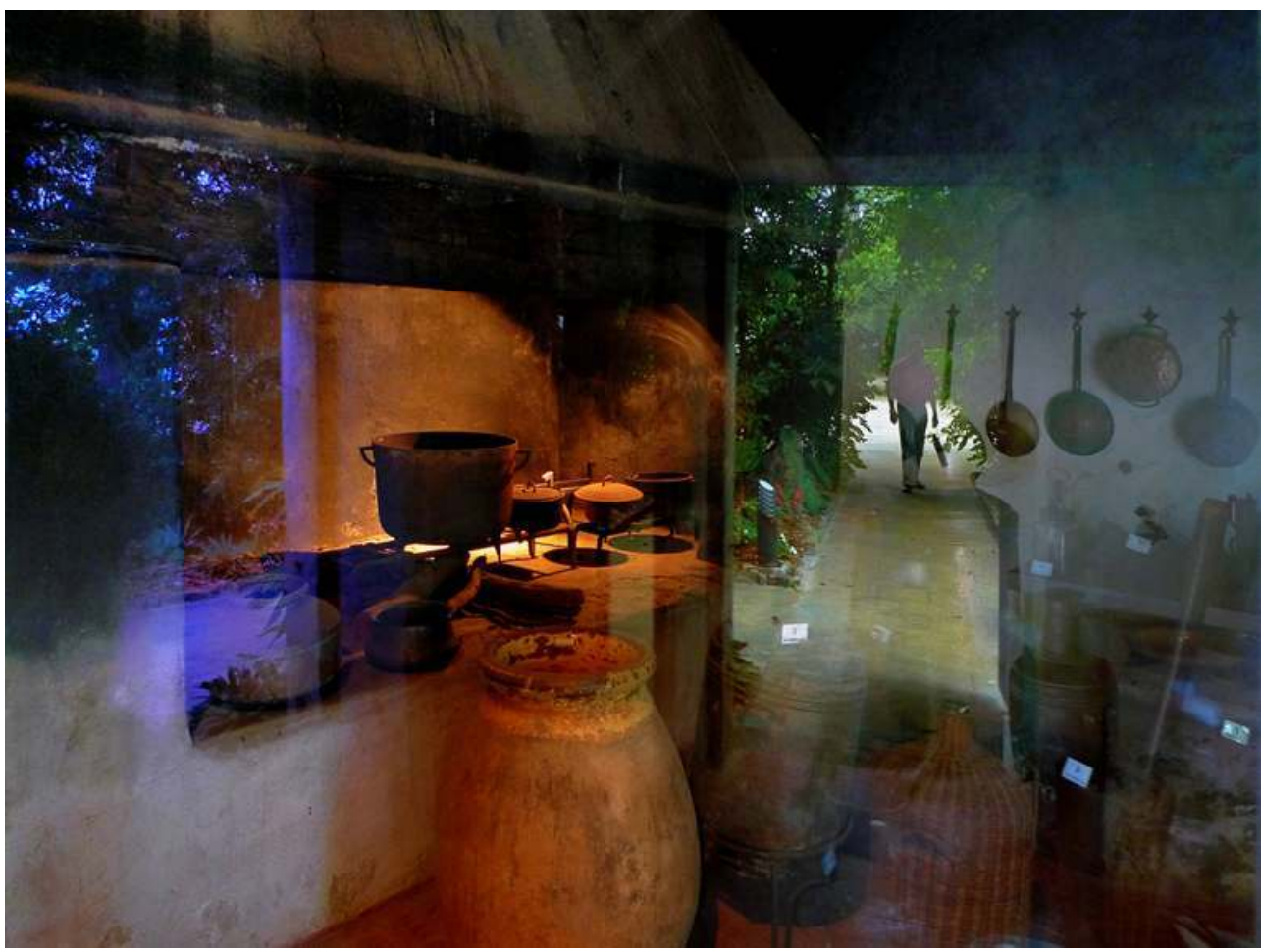

Vue intérieure de la cuisine, photographie en 2010.

(c) Musée historique de Villèle / Raymond Barthe.

Un pavillon en bois recouvert de bardeaux (fig. $\mathbf{n}^{\circ} \mathbf{1 3}$ ), sans doute l'ancien logement du régisseur, a été transformé en espace d'accueil des visiteurs et abrite également un bureau ainsi qu'une salle tenant lieu de réserve pour les collections du musée. 
Figure 13

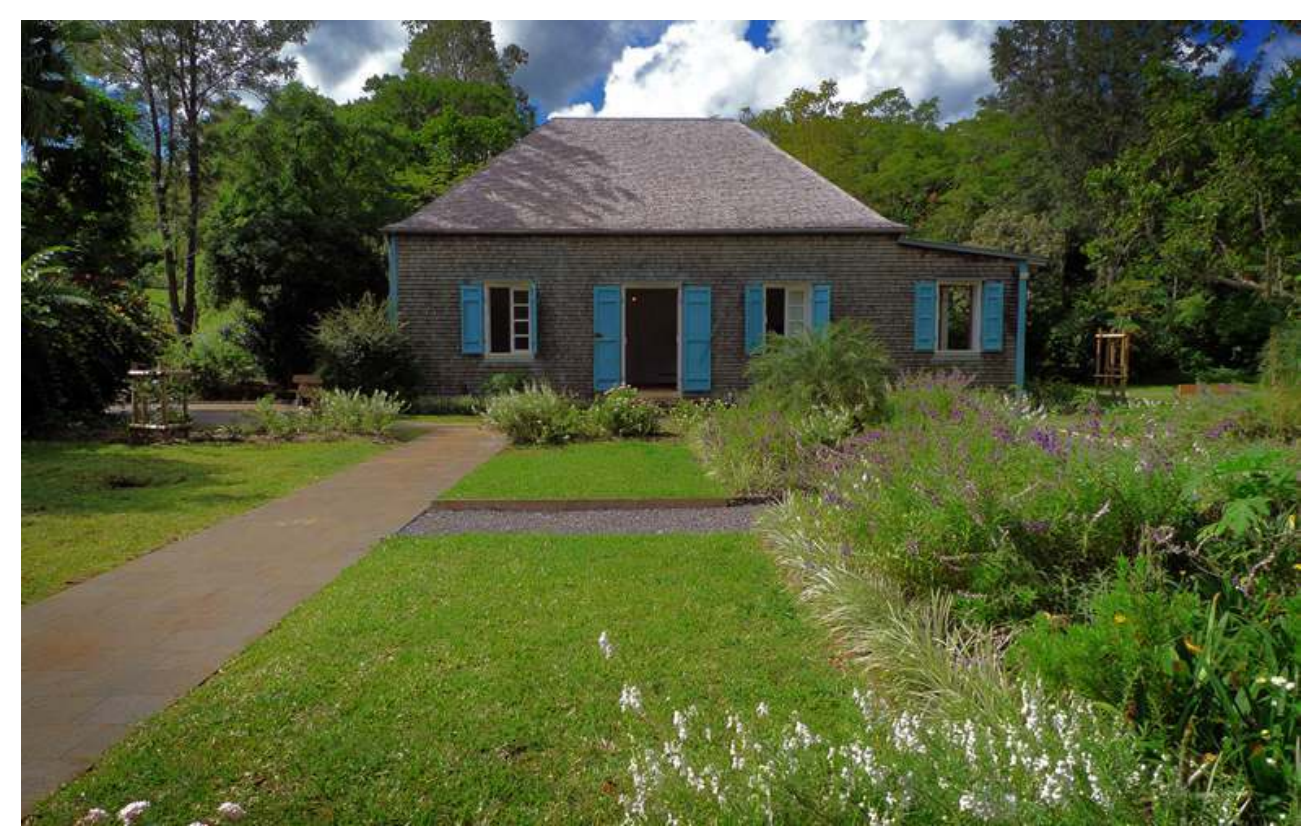

Pavillon d'accueil du musée historique, photographie en 2010.

(c) Musée historique de Villèle / Raymond Barthes.

41 Sur le domaine se trouve un hôpital, bâtiment en pierre construit pour y soigner les esclaves et qui servit de dispensaire jusqu'au début $\mathrm{du} \mathrm{XX}^{\mathrm{e}}$ siècle pour les gens résidant dans le quartier. Ils y étaient soignés par les derniers membres de la famille qui ont vécu sur cette propriété jusqu'en 1973 ! C'est dans ce lieu qu'un mémorial (fig. $\mathbf{n}^{\circ} \mathbf{1 4}$ ) fut mis en place et inauguré en 1996 afin d'honorer les esclaves de la plantation, cafres, malgaches, indiens et créoles. Il s'agit d'une installation créée à partir d'un document d'archives (feuille de recensement) faisant apparaître les noms attribués par les maîtres, l'âge, l'origine ethnique et les fonctions des esclaves. Deux ans avant la célébration du $150^{\mathrm{e}}$ anniversaire de l'abolition de l'esclavage, il était en effet important - par un geste symbolique - de marquer la présence des esclaves sur la propriété. 
Figure 14

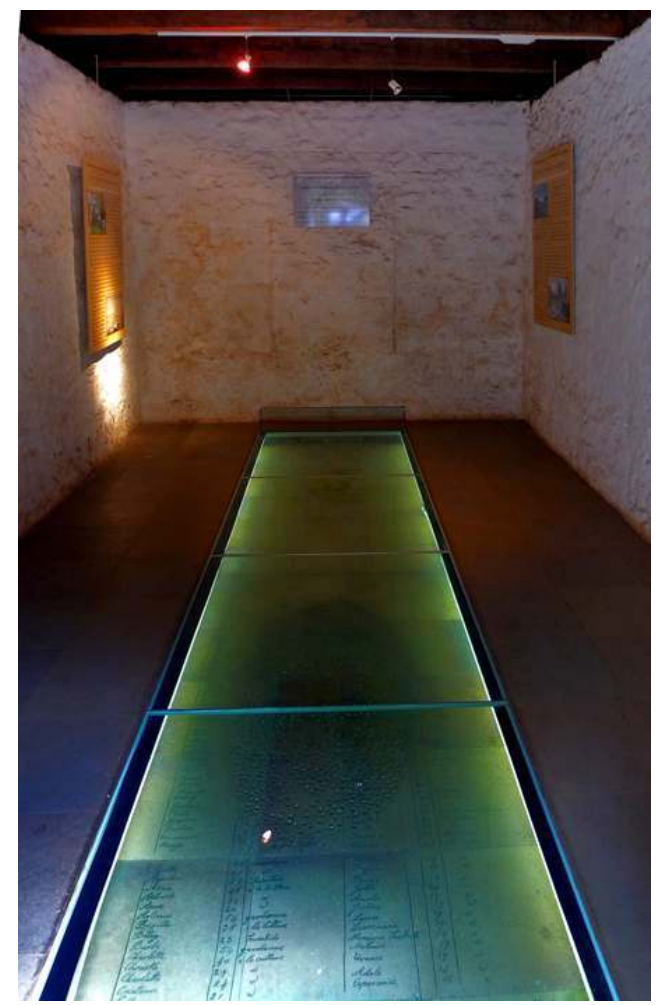

Mémorial dans l'ancien hôpital des esclaves, photographie en 2010.

(c) Musée historique de Villèle / Raymond Barthes.

Les anciens magasins forment un corps de bâtiment tout en longueur et composite. Appelé longère, il abrite de façon sommaire dans huit pièces plus ou moins bien aménagées différents services du musée. Il n'est donc pas accessible aux visiteurs mais il est prévu de réhabiliter l'ensemble du bâtiment afin d'ouvrir de nouvelles salles d'exposition consacrées aux conditions de vie des esclaves sur la plantation, au marronnage et aux abolitions de 1794 et 1848.

43 Au sud de la maison de maître, il reste les ruines d'une usine à sucre construite à l'origine dans les années 1823-24 et considérée par les contemporains comme une usine modèle, équipée d'un moulin à vapeur pour le quartier de Saint-Paul (fig. $\left.\mathbf{n}^{\circ} \mathbf{1 5}\right)$. Le site a fait l'objet d'une campagne de restauration menée dans le cadre d'une action d'insertion de 1993 à 1995. Il constitue aujourd'hui encore une zone peu accessible. Un projet de mise en place de parcours de visite est à l'étude afin de restituer au public l'histoire de la sucrerie tant du point de vue des techniques de fabrication du sucre de canne que de l'organisation sociale des travailleurs. 


\section{Figure 15}

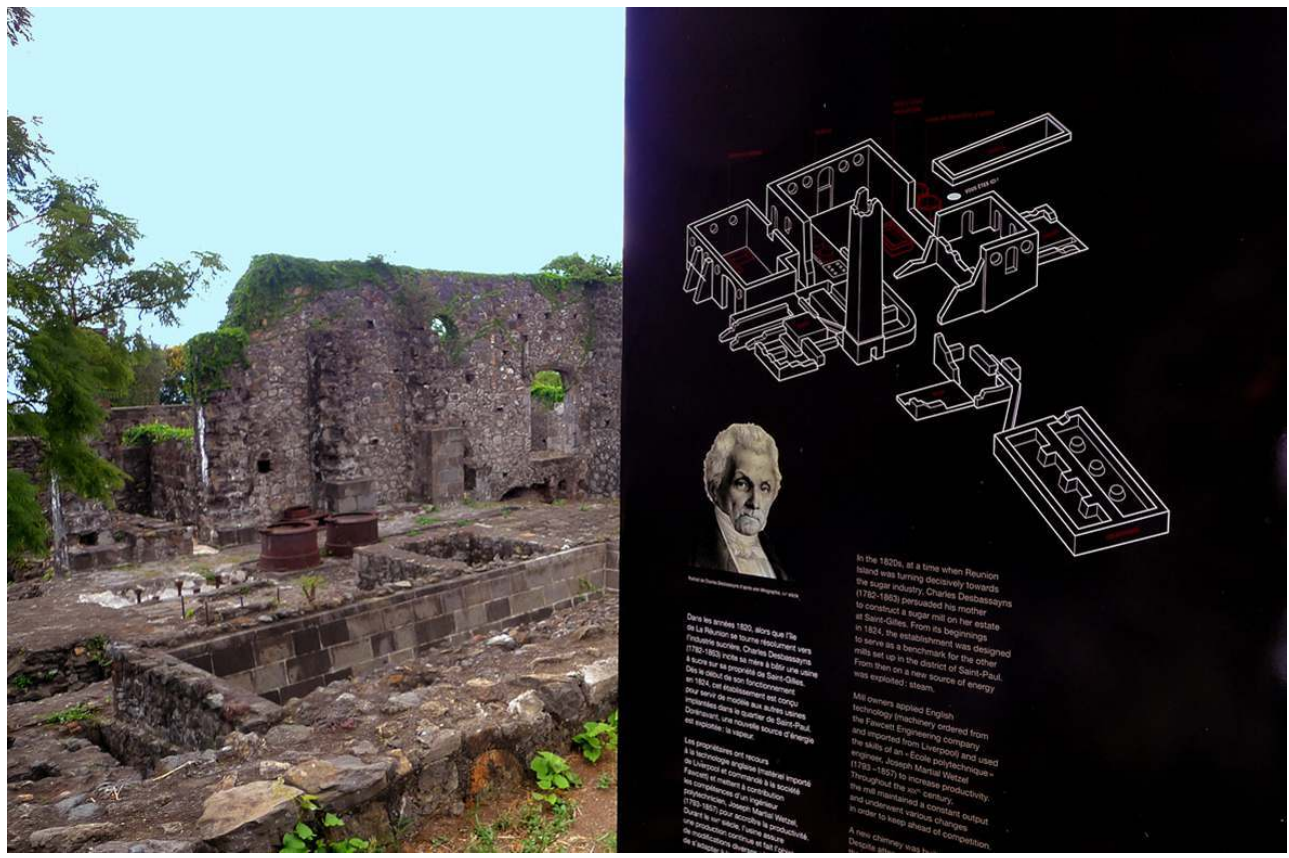

Ruines de la sucrerie Desbassayns, photographie en 2010.

(c) Musée historique de Villèle / Raymond Barthes.

44 Sur le site est érigée une chapelle domestique appelée chapelle Pointue, construite par Madame Desbassayns pour les esclaves. Le premier bâtiment élevé à partir de 1841 offrait une architecture originale d'inspiration néogothique. La chapelle fut détruite par un cyclone en 1932 (fig. $\mathbf{n}^{\circ} 16$ ) et reconstruite dès l'année suivante. Une campagne de restauration en 2002-2003 a permis de restituer son décor intérieur qui n'avait pas été pris en compte lors de la reconstruction de l'édifice en 1933. 
Figure 16

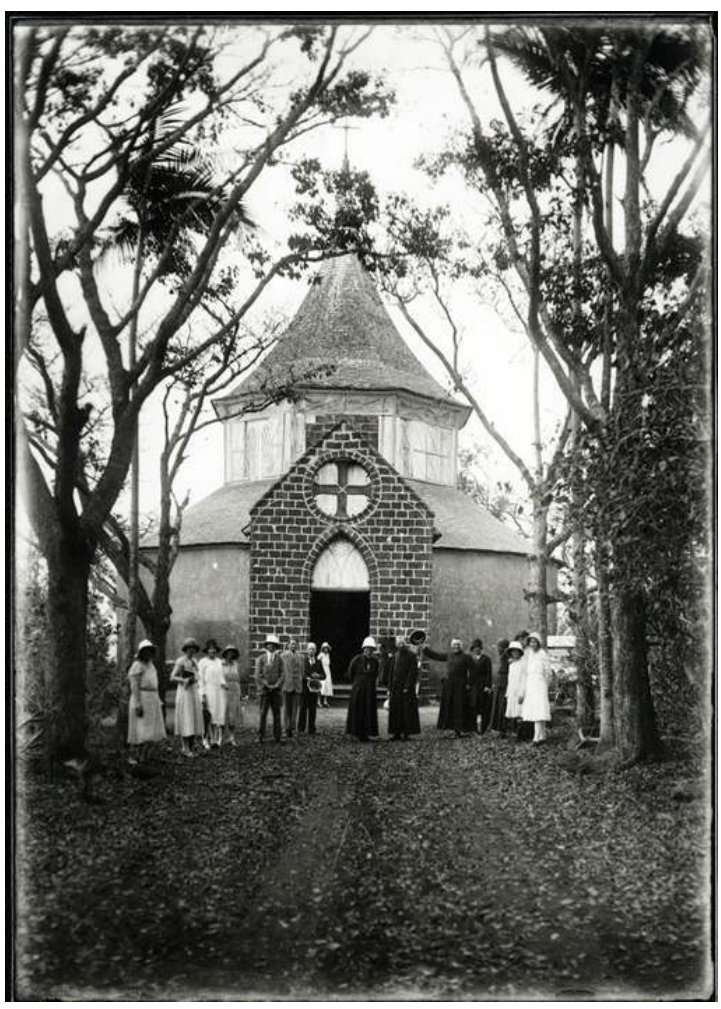

Façade de la Chapelle Pointue, photographie vers les années 1930.

(c) Musée historique de Villèle.

Il est important de signaler l'emplacement du camp, à l'extérieur du musée, où étaient regroupées les paillotes qui abritaient les travailleurs, esclaves puis engagés. Aujourd'hui l'ancien camp, devenu un village résidentiel (fig. $\mathbf{n}^{\circ} \mathbf{1 7}$ ), a conservé peu de traces matérielles de son histoire hormis toutefois l'implantation des maisons les plus anciennes sur des terrains divisés en parcelles, et le réseau des voies de circulation. En revanche, une partie de la population de ce village, constituée par les descendants d'esclaves ou d'engagés, garde des souvenirs très contrastés des anciens propriétaires, surtout des derniers membres de la famille Villèle. Les personnes qui rompent la loi du silence - pour nombre d'entre elles, le passé reste encore un sujet tabou - expriment envers les anciens maîtres du domaine tantôt un fort ressentiment tantôt un respect sans faille, teinté de regrets nostalgiques. Sans doute la position des uns ou des autres dépend-elle de la fonction qu'occupait un membre de leur famille au sein de la gestion du domaine. 


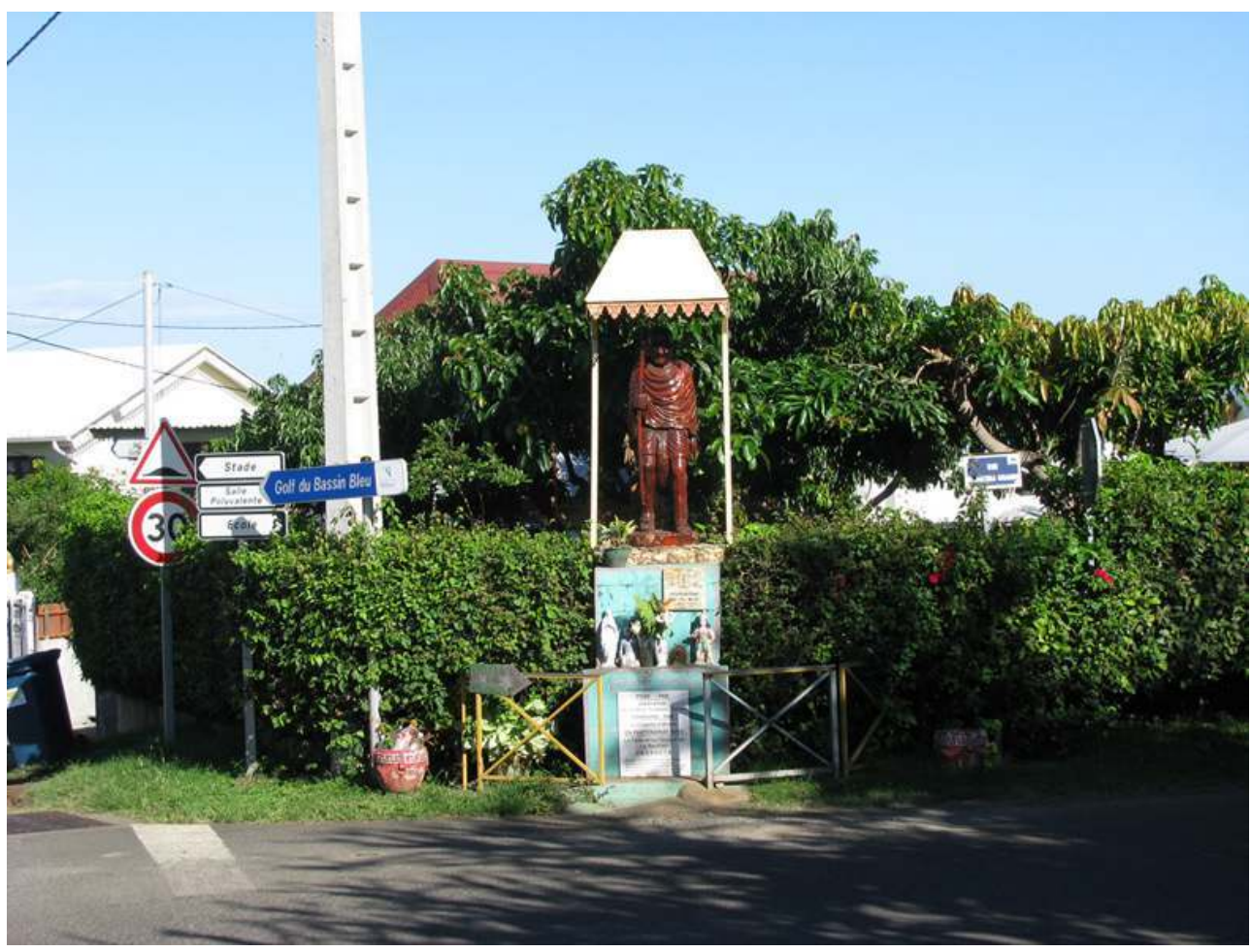

Entrée du village de Villèle, ancien camp d'esclaves, photographie en 2011.

(c) Musée historique de Villèle / Jean Barbier.

\section{Les collections}

Conforme à son statut, le musée de Villèle se définit aussi par ses collections qui constituent autant de supports des connaissances. Jusque dans les années 1980, il faut reconnaître que les collections du musée forment un fonds d'objets et de documents plus ou moins cohérent évoquant différents aspects de l'histoire de l'île, mais sans traiter réellement la question de l'esclavage qui, rappelons-le une fois encore, a laissé peu de traces matérielles.

Les collections sont réparties en trois ensembles : un fonds constitutif, des dépôts et des acquisitions.

- Un premier fonds fut constitué lors de la création du musée en 1974. Un ensemble d'objets d'art décoratif et de mobilier furent acquis par le Conseil général à la famille et permettent jusqu'à ce jour, dans le cadre d'une exposition permanente, d'évoquer au rez-de-chaussée de la demeure familiale le cadre de vie d'une famille de planteurs créoles (fig. $\mathbf{n}^{\circ} \mathbf{1 8}$ ). Le cadre de vie des maitres certes, mais non point celui des esclaves! 
Figure 18

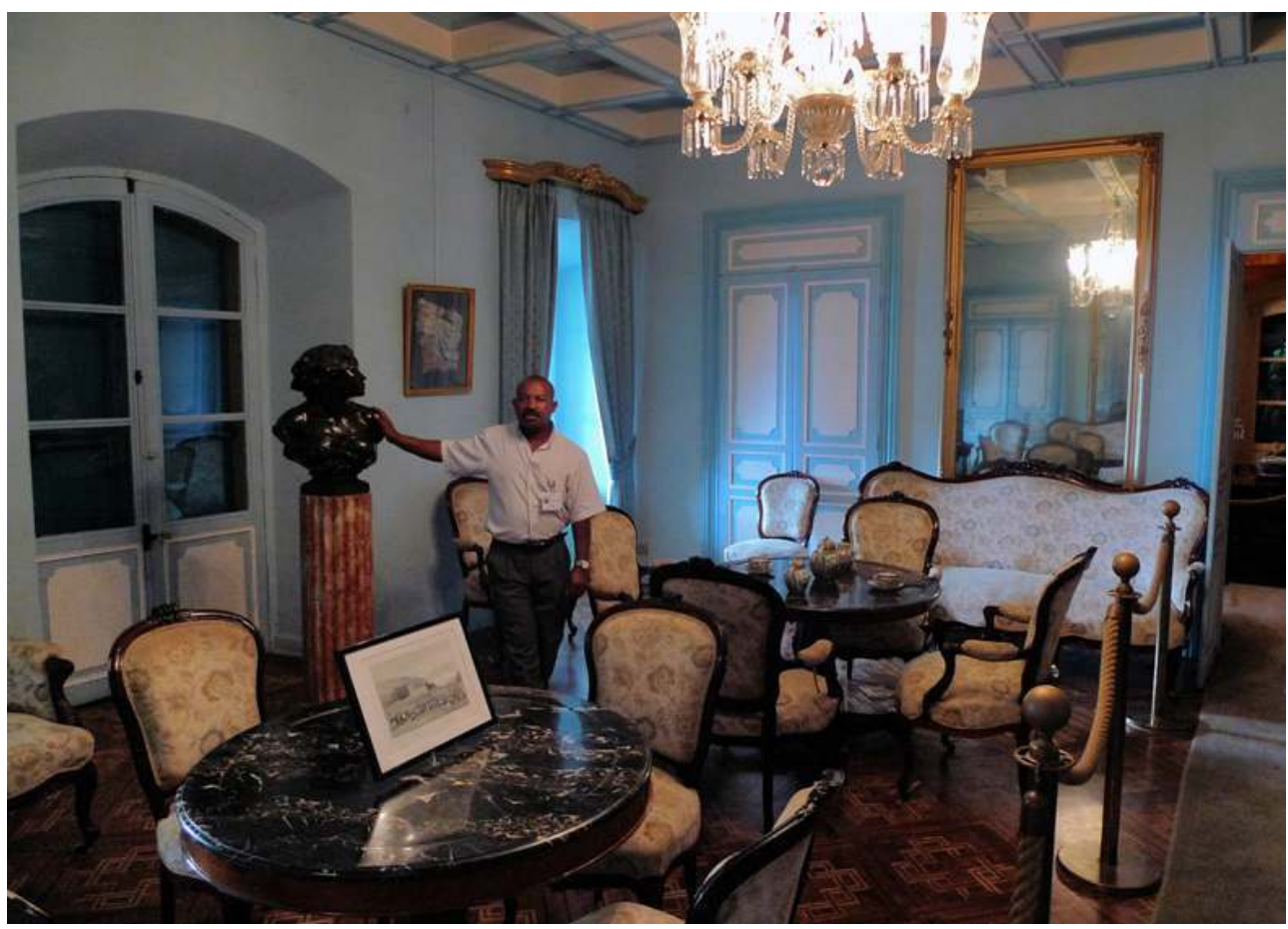

Maison Desbassayns, le grand salon avec un des guides du musée, photographie en 2010.

(c) Musée historique de Villèle / Raymond Barthes.

- Le deuxième ensemble correspond au dépôt d'une partie des collections constituées en vue de l'ouverture d'une section historique envisagée au musée Léon Dierx, lors de sa création en 1911. Les objets collectés, donnés par les grandes familles réunionnaises, avaient été relégués dans l'ombre des réserves de ce musée depuis 1947, date d'arrivée des 157 tableaux d'art moderne de la donation faite par Lucien Vollard. Il est à noter que parmi ces objets se trouve un fusil à pierre offert au redoutable chasseur de noirs marrons (esclaves fugitifs), le dénommé Mussard, bien connu des Réunionnais.

- Les acquisitions faites pour le musée concernent à la fois des collections diverses constituées en vue de la création d'un musée historique dans les années 1970 : des maquettes de navires de la Compagnie des Indes, de la numismatique (trésors trouvés sur l'île) ou bien des porcelaines de la Compagnie des Indes données par une association créée pour le développement des Musées d'Outre-mer (ADMOM). Ces collections devaient témoigner de l'expansion coloniale française.

- C'est à partir des années 1990 que le musée de Villèle oriente sa politique d'acquisition dans plusieurs directions. En premier lieu, il convenait de renforcer les fonds existants, notamment la section historique, par l'achat de documents iconographiques et d'ouvrages concernant plusieurs thèmes, l'expansion coloniale française, la société de plantation mais aussi la traite et l'esclavage à La Réunion (fig. $\left.\mathbf{n}^{\circ} 19\right)$ et dans les pays et îles de l'océan Indien (fig. $\mathbf{n}^{\circ} 20$ ). 


\section{Figure 19}

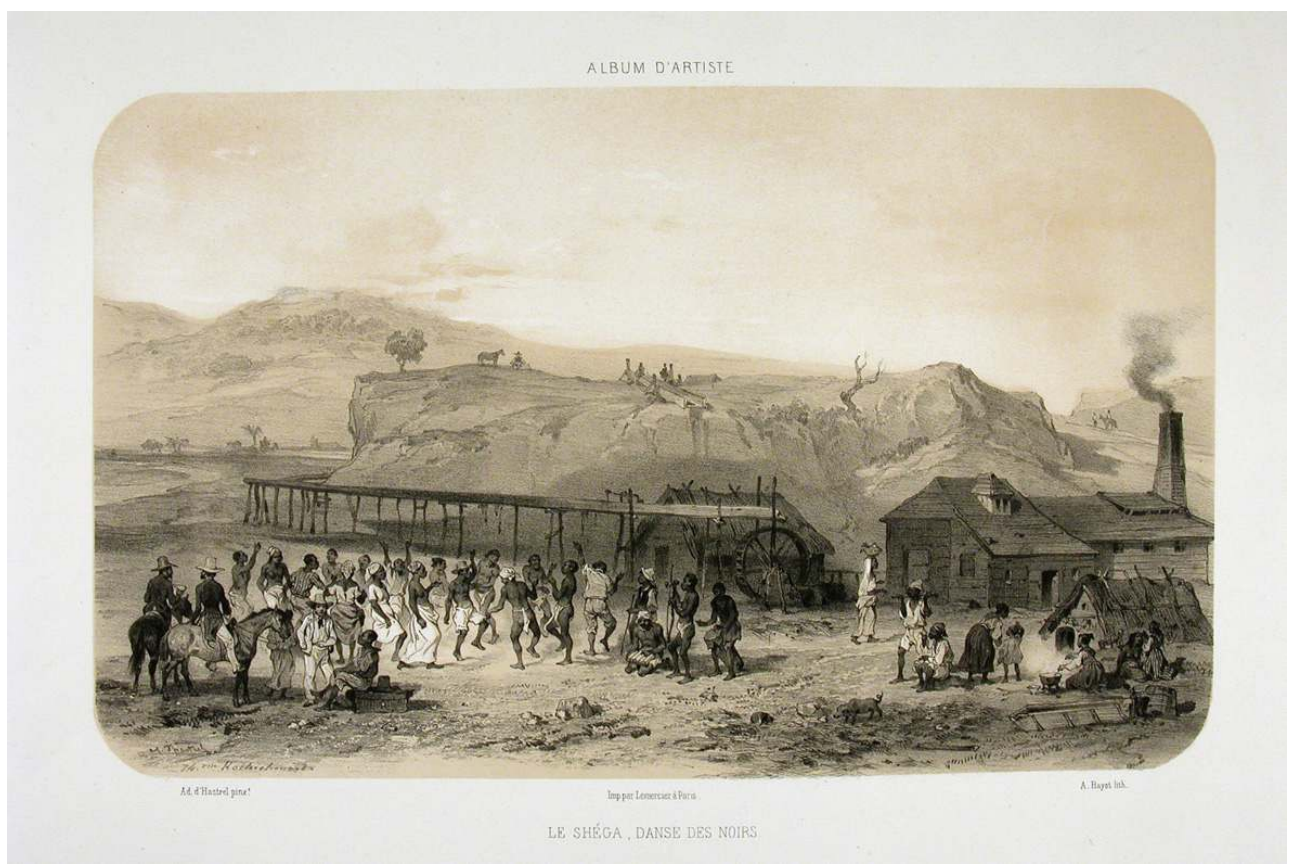

Le shega, danse des Noirs (aujourd'hui appelée maloya), lithographie de 1847.

(c) Musée historique de Villèle. 
$\mathrm{y}$ a des jours où l'on ne trouve rien, me dit le Ma- | vre. En fait d'animaux de basse-cour, on ne voit que

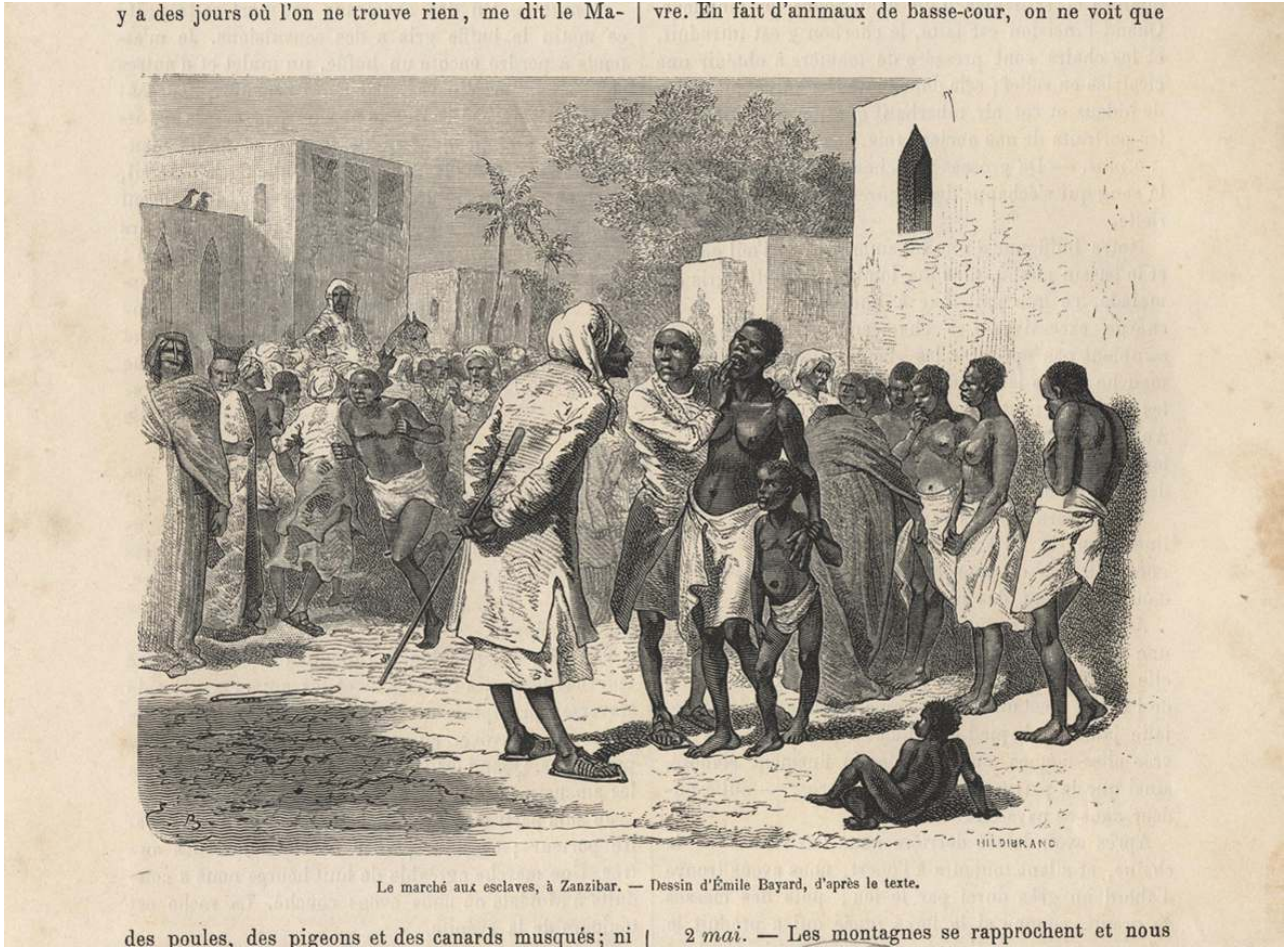

Le marché aux esclaves à Zanzibar, gravure de 1872

(c) Musée historique de Villèle.

- La création de nouveaux fonds a permis notamment à l'art contemporain d'entrer dans les collections du musée de Villèle afin d'évoquer certains aspects de l'histoire de la servitude ou de permettre une approche des cultures des pays de la zone qui ont contribué au peuplement de La Réunion. Ont été ainsi acquises des œuvres picturales de Tanzanie représentatives du mouvement Tingatinga (fig. $\mathbf{n}^{\circ} \mathbf{2 1}$ ), des dessins caractéristiques de la tradition Madhubani (région du Bihar au nord de l'Inde), des sculptures d'artistes Makonde du Mozambique, une collection de tirages du photographe Dityvon réalisés au cours d'un séjour à Zanzibar, des œuvres d'artistes réunionnais ou travaillant sur l'île, Wilhiam Zitte, Antoine Du Vignaux (fig. $\mathbf{n}^{\circ} \mathbf{2 2}$ ), Ann Marie Valencia, Marie-Chrystine Miara, Nelson Boyer... 
Figure 21

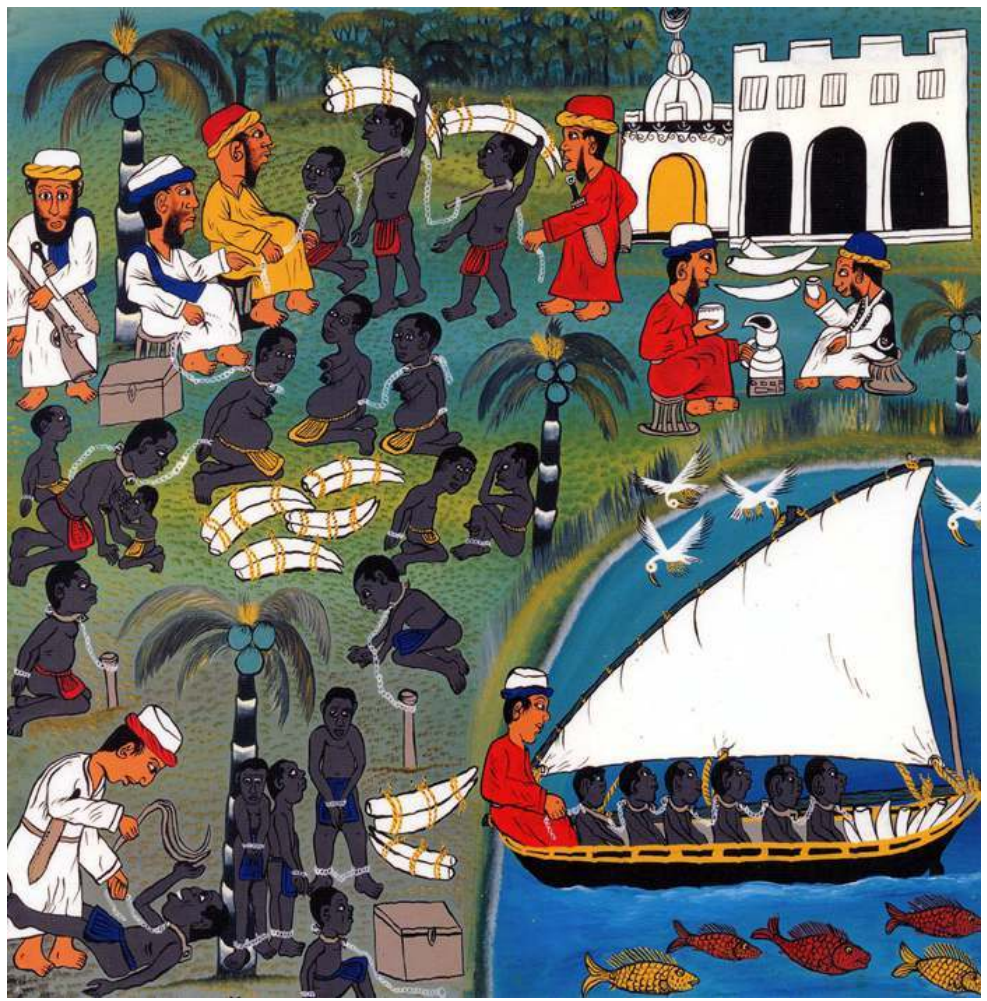

Scène de traite vue par le peintre tanzanien Mohamedi Wasia Charinda, laque sur toile, 1998. (c) Musée historique de Villèle / Jacques Kuyten. 
Figure 22

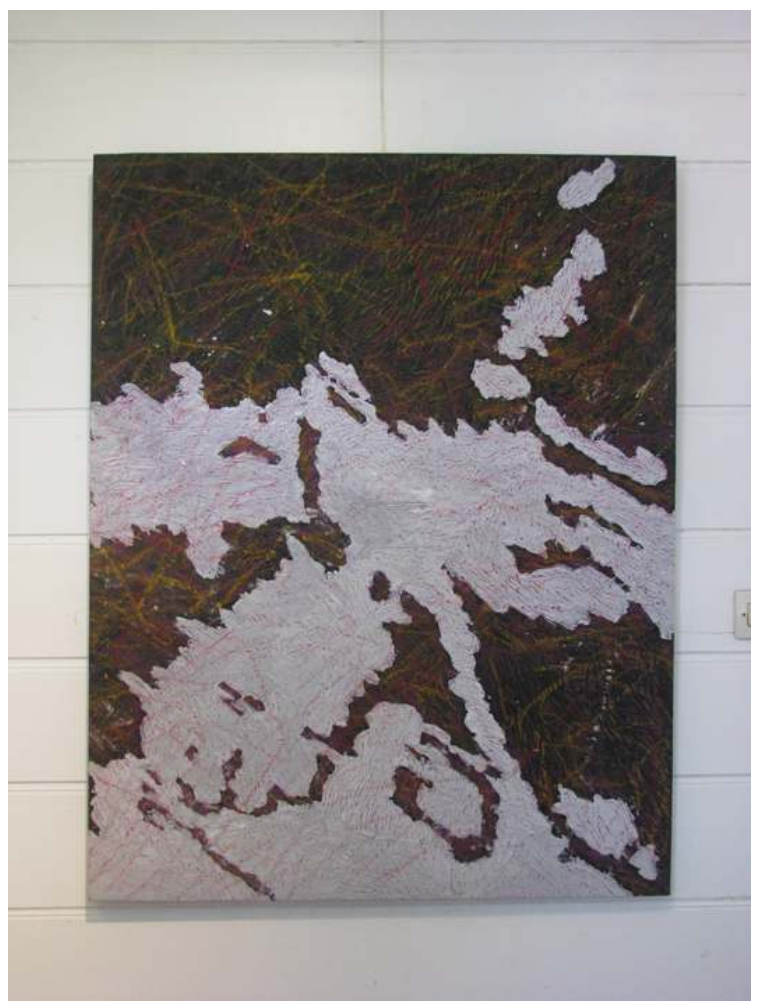

Mains liées, huile sur toile de jute (goni) d'Antoine du Vignaux, 1993.

(c) Musée historique de Villèle.

- Enfin, en 1993, le musée a entrepris la sauvegarde de parties de machines et d'outils de l'ancienne sucrerie (fig. $\mathbf{n}^{\circ} \mathbf{2 3}$ ) disséminés sur la propriété ou trouvés lors des différentes campagnes de déblaiement réalisées à l'occasion d'un chantier de restauration mené sur le site de l'usine. Ce matériel archéologique a fait l'objet d'un recensement, de la constitution d'un fichier d'identification, de la réalisation de croquis et de prises de vues. Une partie des objets de cette collection doit bénéficier d'une importante campagne de restauration qui débutera à la fin de l'année. 


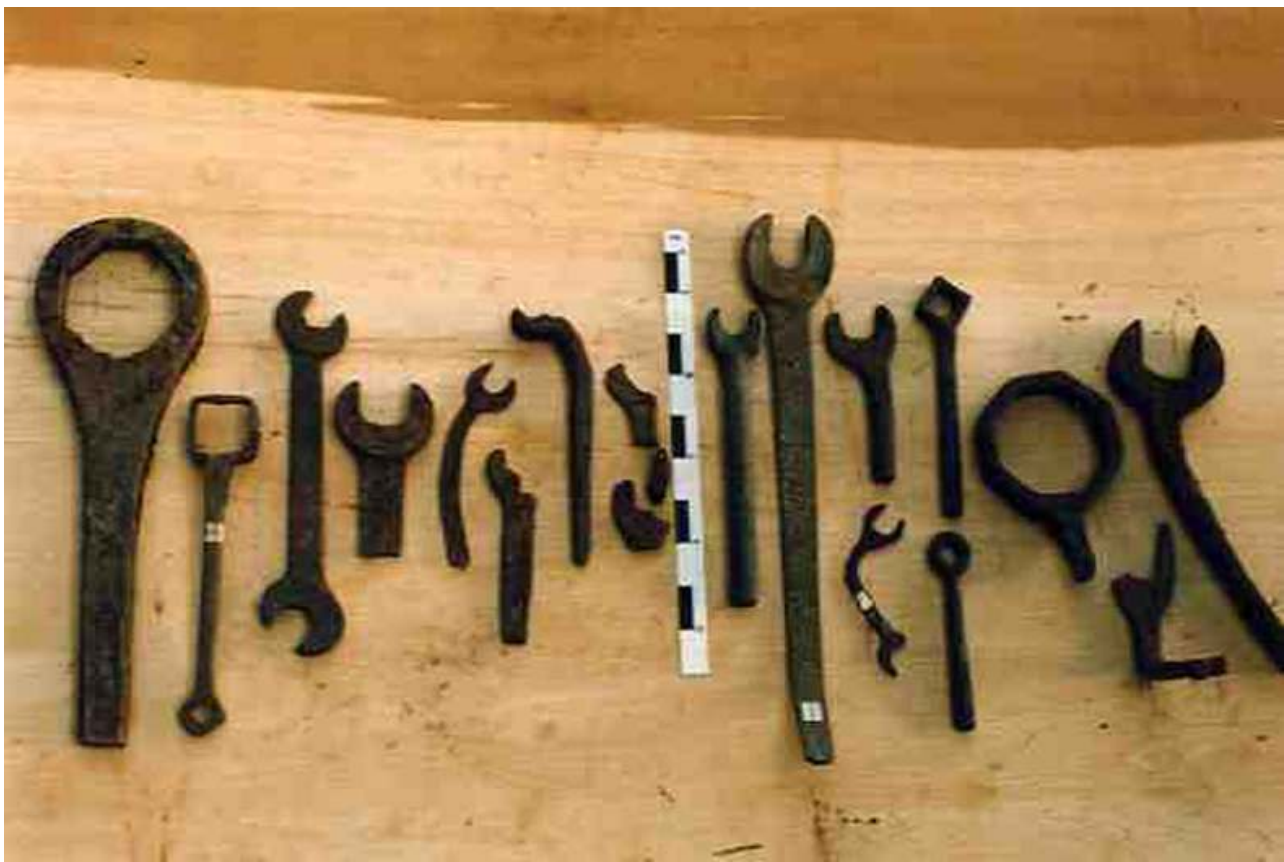

Outils trouvés dans l'ancienne sucrerie Desbassayns, photographie en 1993.

(c) Musée historique de Villèle / Olivier Rouchon.

\section{Les actions du musée pour la diffusion des connaissances}

Depuis les années 1990, le musée historique de Villèle s'est doté de moyens diversifiés afin d'assurer sa mission d'éducation et de transmission des savoirs. Sans développer plus avant le contenu des actions programmées et les modalités mises en œuvre pour chacune d'entre elles, on peut citer quelques axes prioritaires :

- L'action culturelle du service éducatif et du personnel d'accueil du musée est déterminante afin d'engager une démarche de médiation auprès des publics scolaires et des centres de loisirs, incluant la production d'outils pédagogiques, l'accueil des équipes d'enseignants des établissements scolaires et la création d'événementiels (fig. $\mathbf{n}^{\circ} \mathbf{2 4}$ ). 


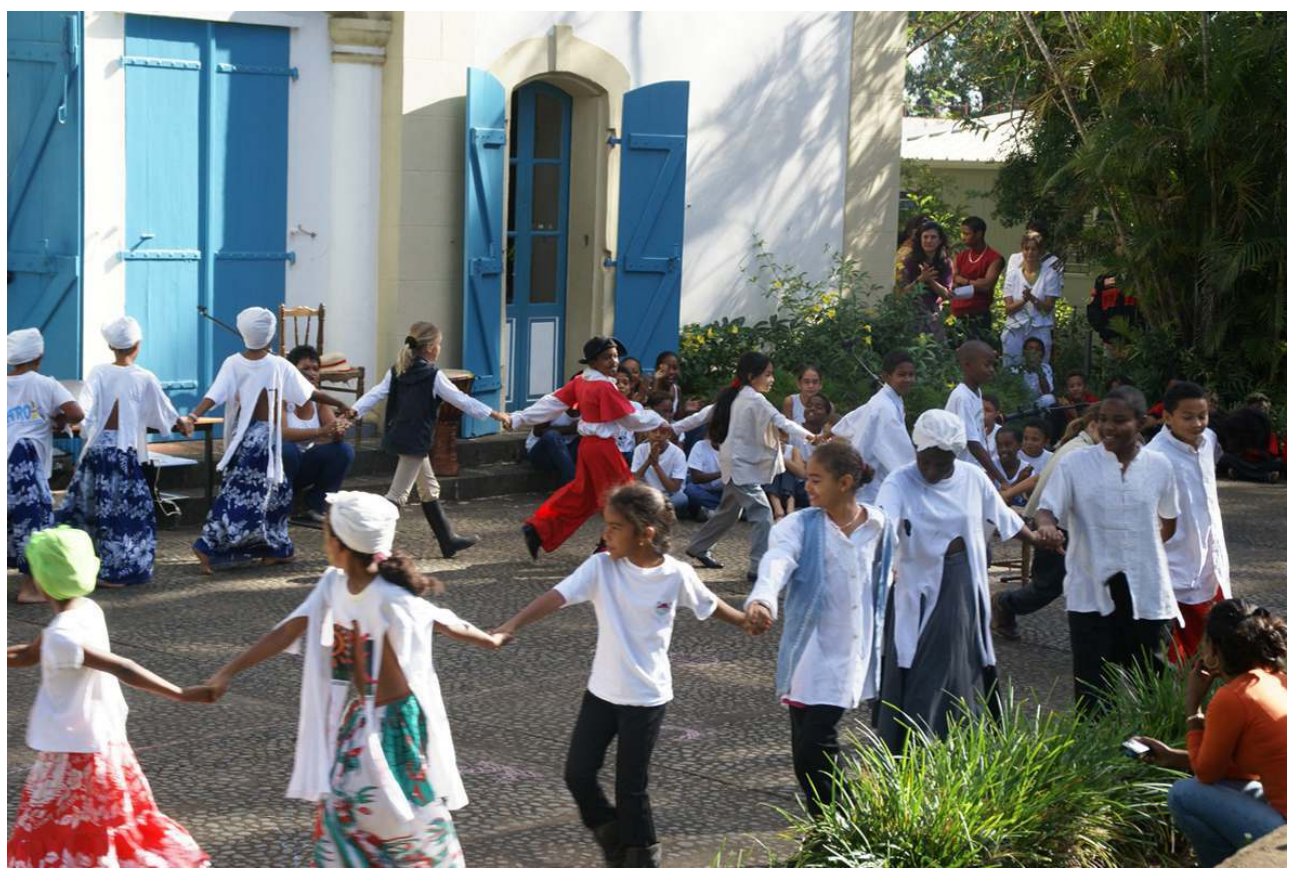

Spectacle d'enfants présenté au musée historique à l'occasion de la Quinzaine des Arts, photographie en 2009.

(c) Musée historique de Villèle / Valéri Sinama.

- La définition d'une politique d'expositions temporaires régulières permet de diversifier l'offre culturelle du musée, de produire également des outils de réflexion et de connaissance, d'enrichir le contenu du parcours muséographique et notamment d'aborder l'esclavage sous différents aspects : recherche généalogique, statut de la femme, conditions de vie, évocation de la « cafritude »...

- Bien que ne disposant pas de surfaces suffisantes, un espace a toutefois été aménagé afin d'organiser un embryon de bibliothèque spécialisée appelée à se développer. Il est constitué essentiellement d'ouvrages sur l'esclavage, l'engagisme et les cultures des pays de la zone océan Indien. En 2011, les fonds se sont enrichis d'un don important de livres anciens sur l'Afrique de l'Est, récits de voyages, expéditions d'explorateurs, missions religieuses.

- Outre la publication des catalogues d'exposition temporaires, le musée historique a lancé cette année une ligne éditoriale intitulée Collection patrimoniale / Histoire. L'objectif est d'assurer une large diffusion des connaissances sur la société réunionnaise apportées par des chercheurs du monde universitaire tout en privilégiant la qualité et la diversité des documents iconographiques conservés dans les institutions patrimoniales, notamment les archives et musées de La Réunion. Le premier titre paru, Henri Paulin Panon Desbassayns. Autopsie d'un gros «Blanc» réunionnais de la fin du XVIII siècle est une étude biographique réalisée par l'historien Claude Wanquet.

- Si le musée historique paraît être le temple de la conservation du passé, il est aussi générateur d'archives du futur. Aussi, la création d'un fonds audiovisuel (fig. $\left.\mathbf{n}^{\circ} 25\right)$ répond à la nécessité d'archiver les temps forts du musée, d'enrichir le discours muséographique notamment à l'occasion de la présentation d'expositions temporaires, de collecter les témoignages d'informateurs, détenteurs de mémoires, de connaissances ou de savoir-faire. 
Figure 25

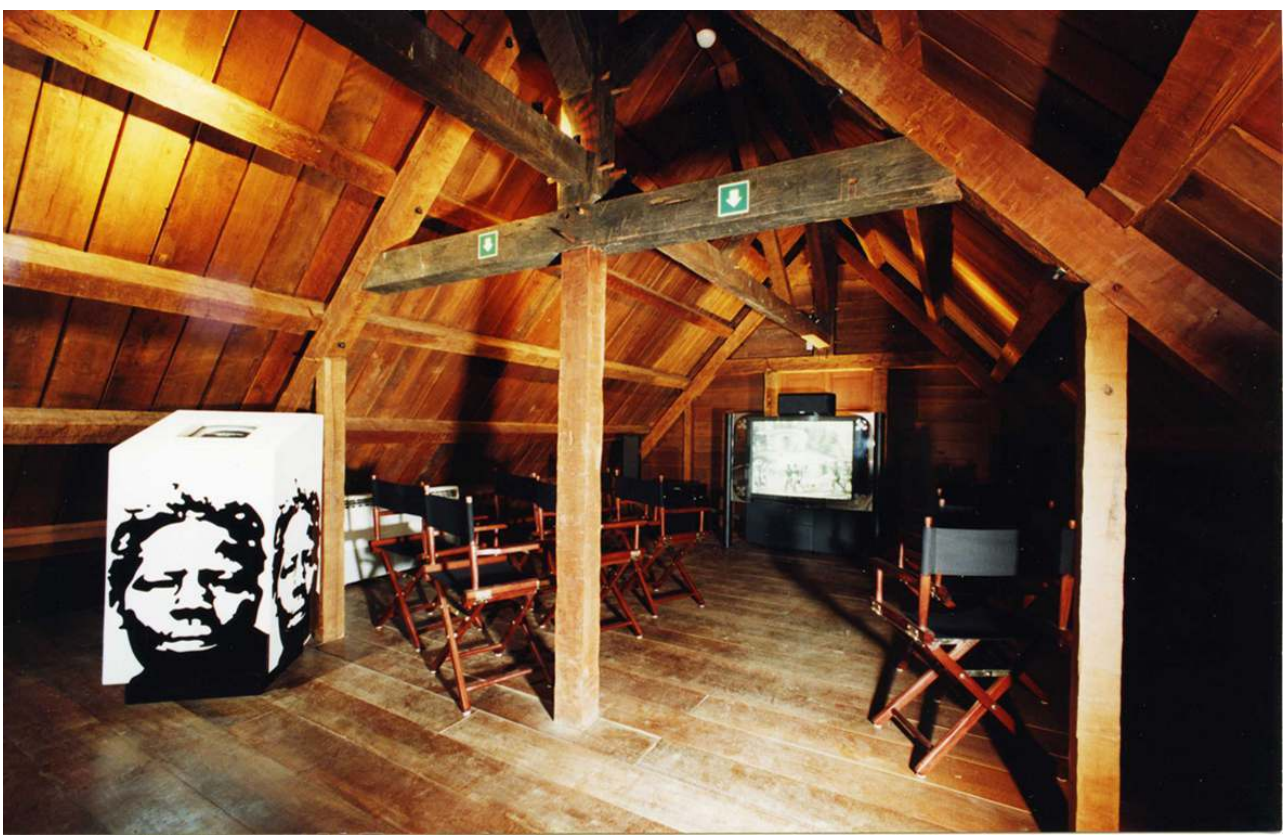

Ancien hôpital des esclaves, salle audiovisuelle, photographie en 1996.

(c) Musée historique de Villèle / Daniel Auguste.

- Des universitaires, historiens, anthropologues, les milieux associatifs (fig. $\mathbf{n}^{\circ} \mathbf{2 6}$ ) sont régulièrement consultés dans le domaine de leurs connaissances afin de prendre part aux projets de recherche du musée de Villèle et constituent véritablement un comité scientifique informel fonctionnant en réseau. 


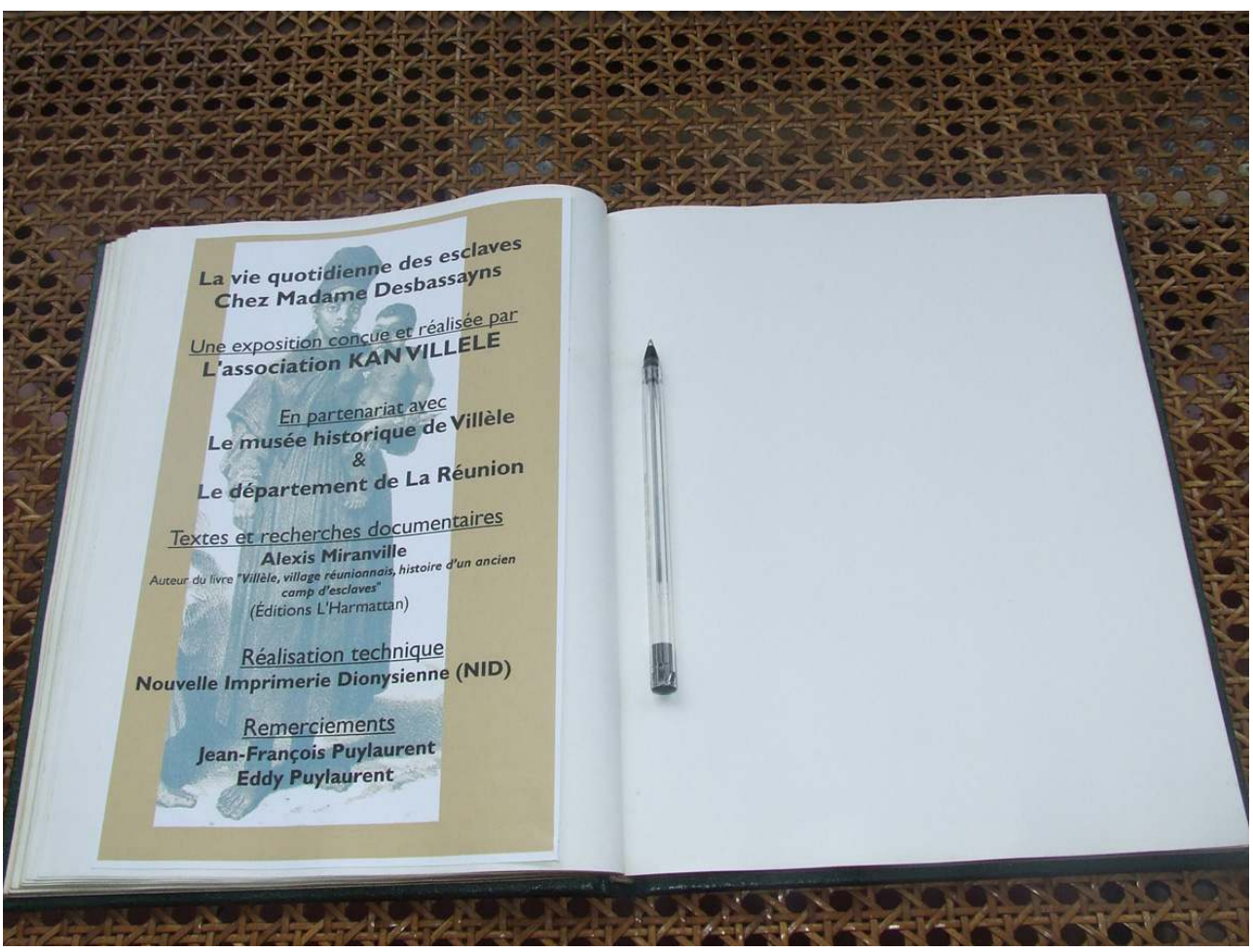

Livre d'or de l'exposition « La vie quotidienne des esclaves chez Madame Desbassayns » organisée par l'association Kan Villèle, photographie le 20 décembre 2007.

(c) Musée historique de Villèle / Association Kan Villèle.

- La signature de conventions internationales avec des institutions culturelles situées dans les pays de la zone indo-océanique est aussi un préalable pour tout projet de coopération. C'est dans ce cadre que le musée historique a pu concevoir respectivement deux expositions: l'une, intitulée Dominique Macondé et présentée à Saint-Gilles-les-Hauts (fig. $\mathbf{n}^{\circ} \mathbf{2 7}$ ), fut le fruit d'un travail scientifique mené en partenariat avec les musées nationaux du Mozambique ; l'autre, opération coproduite en 2010 avec l'Institut Français de Pondichéry, permit de présenter au public indien les liens ayant existé entre l'Inde et La Réunion au travers de l'histoire de l'engagisme. 
Figure 27

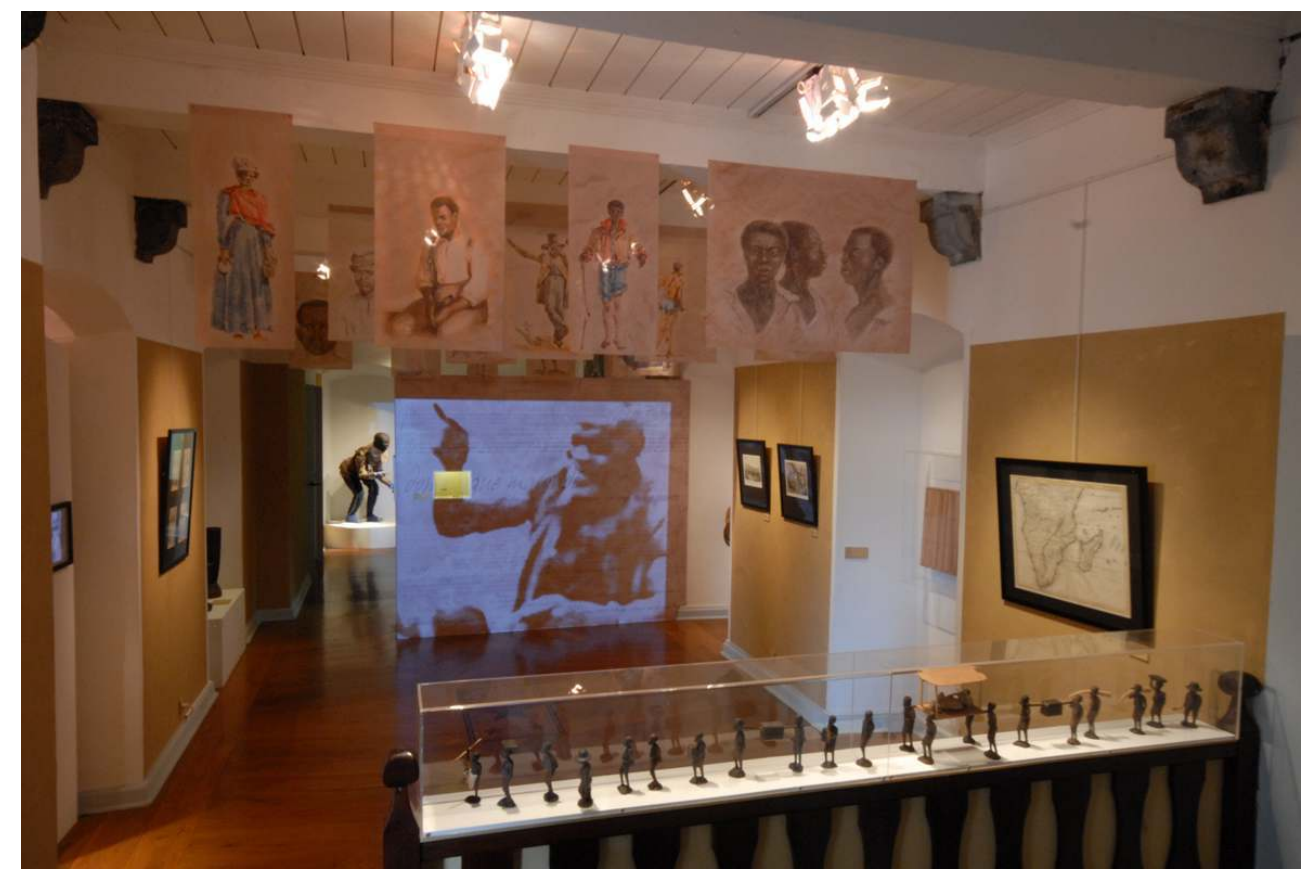

Exposition Dominique Macondé. Mozambique - La Réunion, Musée historique décembre 2006septembre 2007, photographie en 2007.

(c) Musée historique de Villèle / Daniel Auguste.

49 Musée de site, musée de collections, musée de l'économie de plantation, musée de découverte des cultures qui ont forgé l'identité de la société créole, le musée de Villèle doit trouver sa voie à partir de toutes ces identités. Aujourd'hui, il ne donne pas à voir de façon explicite l'histoire de l'esclavage (fig. $\mathbf{n}^{\circ} \mathbf{2 8}$ ) mais doit en ce sens affirmer plus nettement cet objectif propre à sa vocation historique. Il relève ainsi le défi de proposer un programme muséographique adapté à la nature de ses bâtiments, qui pourront ainsi livrer les zones d'ombre de leur histoire. Sans être le lieu exclusif de l'histoire de La Réunion et sans en revendiquer le monopole, il en est l'un des témoins les plus emblématiques et, a fortiori, reste celui, incontournable, de l'esclavage remémoré. 


\section{Figure 28}

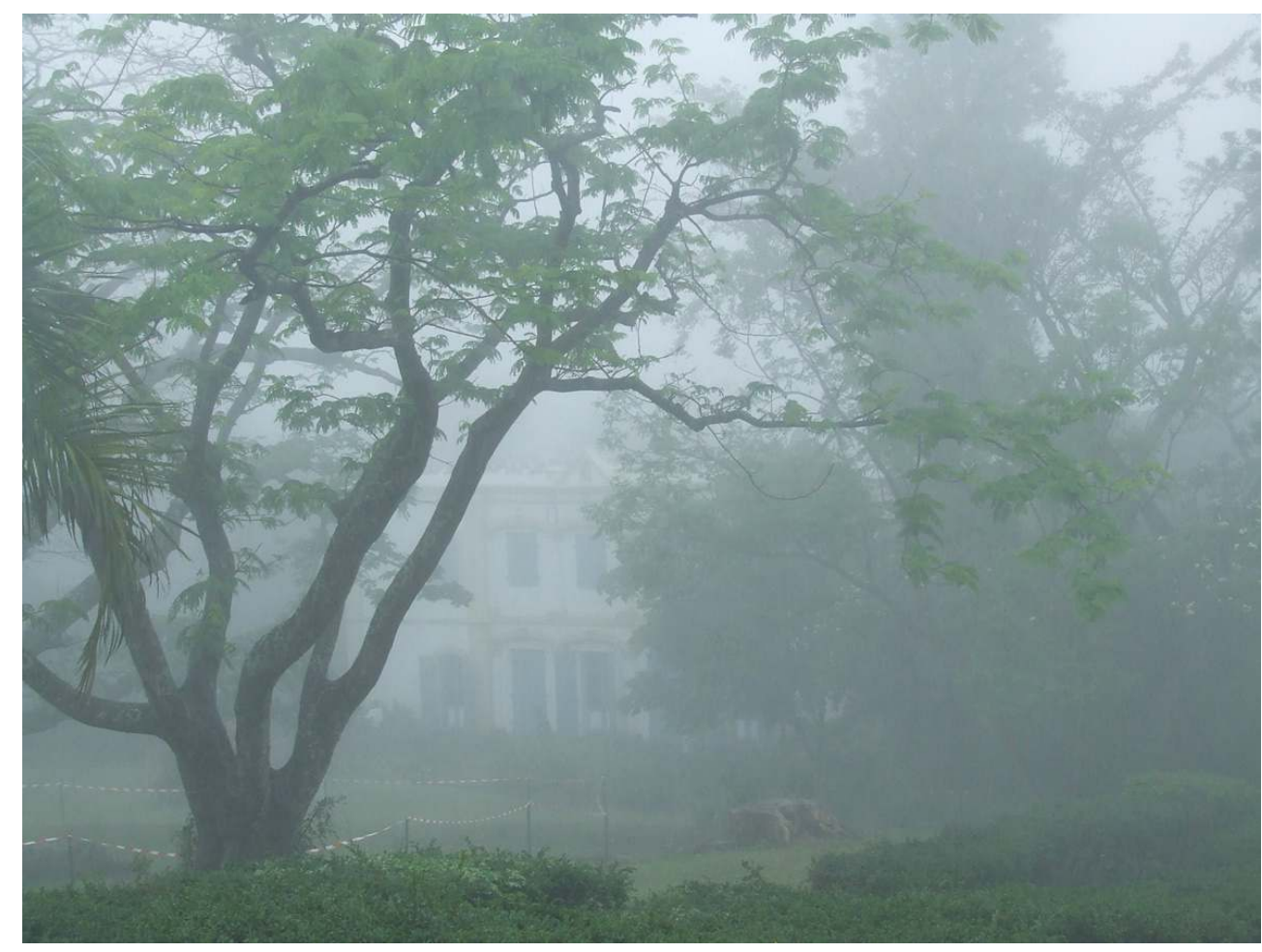

Musée historique, jour de la fête de l'abolition de l'esclavage, photographie le 20 décembre 2008.

(c) Musée historique de Villèle / Suzelle Boucher.

\section{NOTES}

1. - Les pas géométriques ou "pas du roi » sont une extension du droit royal sur les rives des cours d'eau et le littoral. Ils représentent les 50 pas du domaine public (81,20 mètres).

\section{RÉSUMÉS}

Inauguré en 1976, le musée historique de Villèle est le premier musée créé après la départementalisation de l'île en 1946. Patrimoine historique, il se présente avant tout comme un lieu incontournable de l'histoire sociale de La Réunion, marquée à la fois par la diversité des populations venues d'Europe, d'Afrique et d'Asie et par le développement d'un système économique basé sur l'exploitation d'une main d'œuvre servile, en majorité des esclaves aux $\mathrm{XVIII}^{\mathrm{e}}$ et XIX ${ }^{\mathrm{e}}$ siècles puis, à partir de 1848 jusqu'au début du XX $\mathrm{X}^{\mathrm{e}}$ siècle, des travailleurs engagés. 
La communication porte sur une présentation succincte du lieu, sur le parti pris et les limites du discours muséographique actuel, sur les actions entreprises pour prendre en compte, développer, évoquer ou expliciter l'histoire de l'esclavage.

Inaugurated in 1976, the Villèle History Museum was the first museum to be created after Reunion Island became a French department in 1946. Today it is a place of reference for the understanding of Reunion social history. This heritage site evokes not only the diversity of those populations hailing from Europe, Africa and Asia but also bears witness to the development of an economic system based on the exploitation of servile labour, mainly slaves during the XVIII ${ }^{\text {th }}$ and $\mathrm{XIX}^{\text {th }}$ centuries and then indentured workers from 1848 until the beginning of the $\mathrm{XX}^{\text {th }}$ century. This paper provides a brief introduction to the site, explores the museum's commitment and the limits of its current message, and also examines the courses of action taken to consider, develop, evoke and explain the history of slavery.

\section{INDEX}

Mots-clés : Océan indien, Bourbon, Réunion, Saint-Gilles-les-Hauts, Saint-Paul, La Grande Chaloupe, Pondichéry, Panon-Desbassayns, Mozambique, Macondé, Madhubani, Tingatinga, Villèle, Zanzibar, habitation, maison de maître, esclave, colon, travailleur engagé, créole, indien, malgache, lieu de mémoire, Compagnie des Indes, lazaret, système colonial, abolition, économie de plantation, planteur, Cafre, Noir, chapelle, usine à sucre, hôpital, camp

Keywords : Indian Ocean, Reunion Island, Pondicherry, Panon Desbassayns, Makondé, Plantation house, owner's house, slave, sharecropper, indentured worker, Indian, Malagasy, place of remembrance, East India Company, colonial system, plantation economy, planter, Black, Chapel, sugar refinery, hospital, compound (slave)

\section{AUTEUR}

\section{JEAN BARBIER}

conservateur territorial du patrimoine en chef, directeur du Musée historique de Villèle musee.villele@cg974.fr/jean.barbier@cg974.fr 